\title{
Spectroscopy of Gauge Theories Based on Exceptional Lie Groups
}

\author{
Philippe Pouliot \\ Physics Department \\ University of Texas at Austin \\ Austin, TX 78712 USA \\ Email: pouliot@physics.utexas.edu
}

\begin{abstract}
We generate by computer a basis of invariants for the fundamental representations of the exceptional Lie groups $E_{6}$ and $E_{7}$, up to degree 18 . We discuss the relevance of this calculation for the study of supersymmetric gauge theories, and revisit the self-dual exceptional models. We study the chiral ring of $G_{2}$ to degree 13 , as well as a few classical groups. The homological dimension of a ring is a natural estimator of its complexity and provides a guideline for identifying theories that have a good chance to be amenable to a solution.
\end{abstract}

Keywords: Supersymmetry, Chiral Rings, Gauge Theories, Duality, LiE, Syzygy, Classical Invariant Theory. 


\section{Contents}

1. Introduction 2

2. Mathematical Preliminaries 3

3. Theories that must have a dual $\quad 4$

$3.1 E_{6}$ with 5 flavors 5

$3.2 \operatorname{Spin}(16)$ with one spinor and two vectors 6

4. Invariants of $E_{6}$ with fundamentals $\quad 7$

4.1 Status of the self-dual model for $E_{6}$ with 6 flavors 9

5. Invariants of $E_{7}$ with fundamentals $\quad \mathbf{1 0}$

5.1 Status of the self-dual model for $E_{7}$ with 4 flavors 16

6. Examples with Other Groups 17

6.1 The ring of $S U(2)$ with fundamentals 17

6.2 Invariants and syzygies of $G_{2}$ with fundamentals $\quad 18$

6.3 Invariants of $S U(3)$ with symmetric tensors 22

6.4 Invariants of $S U(4)$ with symmetric tensors 23

6.5 Invariants of $S U 5)$ with symmetric tensors 24

$\begin{array}{ll}\text { 7. Conclusion } & 24\end{array}$

A. The constraints of degree 18 for $E_{6} \quad 25$

B. Some glueballs of $E_{6} \quad 26$

C. Computer Program Code for LiE 28 


\section{Introduction}

In the past several years, much progress has been made in understanding the behavior of supersymmetric theories at low energy. In the best of cases, weakly coupled dual descriptions have been found for strongly coupled supersymmetric theories, which is tantamount to an exact solution of the theory in the very low energy regime and for very large distances ${ }^{1}$.

However, many key questions have not been answered. For example, given a gauge group and matter content superfields in some representation of the gauge group, what is the low energy behavior of the theory? For starters, most theories are free in the low energy regime since they are not asymptotically free. Of those that are asymptotically free, most live in an interacting non-Abelian Coulomb phase. A smaller fraction have extended supersymmetry or live in a confining phase or an Abelian Coulomb phase at low energies, and are rather well understood. But the behavior of the majority of theories in a non-Abelian Coulomb phase remains to be understood. Although not of much direct physical interest, it is an important mathematical physics problem.

An important insight into the behavior of theories in a non-Abelian Coulomb phase is duality [1]. It is unclear whether duality is generic or a feature of a few especially simple theories. Thus a great deal of work was done and has led to finding many more examples of duality beyond the examples of [1] for the classical groups $S U, S O$ and $S p$ with matter fields in copies of the fundamental representations. The simplest examples of duality arise when the theory confines and the low energy dual description just consists of gauge invariant mesons and baryons [2]-[22] and there is a claim that the theories in a confining phase have all been found and studied, at least for simple gauge groups $[15,16]$. When the dual theory is not confining, there are numerous examples for the classical groups with a variety of features: terms in the electric superpotential, tensor products of groups, (anti)-symmetric or adjoint representations, finite theories, [23]-[42], while for the exceptional groups, there is a family of examples based on $G_{2}$ and the Spin groups [43]-[47] and isolated examples of a so-called "self-duality" [48]-[52].

The battery of tests that known dualities pass are also the tools being used to search for new examples. Several of these tests require detailed knowledge of the theory being studied. This is the case for matching the flat directions, matching the spectra or the

\footnotetext{
${ }^{1}$ For practical reasons, we will arbitrarily limit our context to $\mathcal{N}=1$ supersymmetric gauge theories in four dimensions, although our results have a more general range of applicability and although there has been considerable work relevant to the issues addressed in this paper coming from string theory, extended supersymmetry, or theories in more or fewer dimensions.
} 
chiral rings, or for checking 't Hooft's anomaly matching conditions [53]. For all these tests, a knowledge of the gauge invariant chiral superfields is necessary.

Among the many impediments in finding more examples, one problem that is quite tractable is obtaining the structure of the chiral rings for theories of interest. In this paper, we extend the amount of data known about such gauge invariant superfields for some specific cases. We focus our effort on the exceptional simple Lie groups $E_{6}$ and $E_{7}$ with matter quarks in many copies of the fundamental representation. We also illustrate with other groups how very detailed information can be obtained, systematically and for any representation of any Lie group, with enough computer power. In the process, we make tools, which are not new, more easily available, for generating this kind of data.

Now a short summary of the contents. In section 2 , we provide a brief mathematical guide and references. In section 3, we mention the existence of 2 theories which must have a dual description, which offers some motivation for the calculations done in this paper. In section 4 , we find an (almost) complete list of the invariants of $E_{6}$ for copies of the 27-dimensional representation, a list of 20 invariants up to degree 18 . In section 5 , we show how this effort stalls for $E_{7}$, by exhibiting several hundred invariants, with many more yet to be found. The present author sees this complexity as a reasonable apology for failing to find the dual descriptions. We then move on to simple examples and recover some known mathematical results. In section 6.1, we study in some detail the syzygy chain for $S U(2)$ with fundamentals; in section 6.2 , we recover the wellknown results for $G_{2}$; in sections $6.3,6.4$ and 6.5 , we find some invariants for copies the symmetric tensor representations of $S U(3), S U(4)$ and $S U(5)$, and observe that the complexity dramatically increases with the rank of the group. We include appendices on constraints and glueballs in $E_{6}$, as well as some computer code for the LiE software that we used to do these calculations. In the conclusion, we mention some obvious extensions to this work, and briefly discuss its applicability.

\section{Mathematical Preliminaries}

Our goal in this paper is to find a minimal list of the "fundamental" polynomial invariants $^{2}$. This is known as a Hilbert basis: a finite collection of invariants $I_{1}, \ldots, I_{m}$ forms a Hilbert basis if every other invariant can be written as a polynomial function of the basis invariants: $I=P\left(I_{1}, \ldots, I_{m}\right)$ ([58], page 39.). An important theorem of Hilbert showed that any finite system of homogeneous polynomials admits a Hilbert basis. The elements of the Hilbert basis are said to be (polynomially) independent.

\footnotetext{
${ }^{2}$ For a section of the mathematical literature relevant to invariant theory: [56]-[70].
} 
There are other, less stringent, notions of independence: the invariants can be rationally, algebraically, or functionally independent. For the purposes of duality for supersymmetric theories in a non-Abelian Coulomb phase, which notion of independence is relevant? At the most basic level of matching flat directions between the electric and the magnetic theories, all that matters is functional independence: that the moduli spaces of vacuum states have the same dimensions and that the theories remain dual along the flat directions. At the level of 't Hooft anomaly matching, functional independence is clearly not enough and perhaps algebraic independence is what one is asking for: if 't Hooft's anomaly matchings are satisfied for a basic set of invariants, they would no longer be satisfied for the invariants obtained by acting with some functions on these invariants. Finally, the best one can impose from the requirement of supersymmetry is a complete isomorphism of the chiral rings $[71,72,73]$. For other theoretical work relevant to these issues, see [74]-[79].

The chiral rings that arise in supersymmetric gauge theories can be very simple or extremely complicated. In ring theory, there is a natural notion of the complexity of a ring, measured by its homological dimension. The invariants typically satisfy constraints, called first-order syzygies. These first-order syzygies themselves satisfy constraints, called second-order syzygies, and so forth. For the rings that we are concerned with here, Hilbert's theorem applies, and this chain of syzygies must terminate. The length of this chain is known as the homological dimension.

When the ring is freely generated, the homological dimension is zero. However, when it is not freely generated, it is typically very large. That these rings are very complicated is known by mathematicians. For example, for irreducible representations other than the fundamental or the adjoint, the homological dimension of $E_{7}$ is known to be larger than 26334 (page 11 of [61])!, with similar surprisingly large lower bounds on the homological dimensions for other exceptional and spin groups. However, in this paper, we are mostly concerned with multiple copies of the fundamental representation. We will find that in these cases too, the homological dimension is likely to be very large.

\section{Theories that must have a dual}

Although this complexity was known to some physicists (cf. page 2 of [71]), it had escaped the author. Most supersymmetric gauge theories exist in a non-Abelian Coulomb phase at long distances. Among them, just a few have the noteworthy feature that a gauge invariant baryon in their spectrum has an $R$-charge that is less than $2 / 3$. Such theories must have a dual description [1], because the spectrum of the electric theory is not in a unitary representation of the superconformal algebra. Furthermore, this baryon of charge less than $2 / 3$ must appear as a fundamental free field in the dual 
description. It is also to be expected, if the known examples can be a guide, that the full dual description will be free. Below, we give examples of such theories that must have duals. Our analysis of these examples is inconclusive, but might possibly be of interest to someone seeking to find duals.

\section{$3.1 E_{6}$ with 5 flavors}

One such example $[54,52]$ is the theory with $\mathcal{N}=1$ supersymmetry and with gauge group $E_{6}$ and five flavors in the 27-dimensional fundamental representation. The global anomaly-free symmetry is $S U(5) \times U(1)_{R}$. In the table below, we list the quantum numbers of the fields.

\begin{tabular}{|c|c|c|c|}
\hline & $E_{6}$ & $S U(5)$ & $U(1)_{R}$ \\
\hline$Q$ & 27 & 口 & $1 / 5$ \\
\hline \hline$I_{1}$ & 1 & Ш & $3 / 5$ \\
$I_{2}$ & 1 & 王 & $6 / 5$ \\
$I_{3}$ & 1 & 田 & $9 / 5$ \\
$I_{4}$ & 1 & 而 & $12 / 5$ \\
\hline
\end{tabular}

We have included the spectrum of polynomially independent gauge invariant baryons. This theory is in a non-Abelian Coulomb phase, since $S U(3)$ along its flat direction is. And because the $R$ charge of $I_{1}$ is less than $2 / 3$, this theory must have a dual.

For future reference, we give the contributions of the various fields to the anomalies:

\begin{tabular}{|c|c|c|c||c|c|c|c|}
\hline Gauge & Field & $S U(5)$ & $U(1)_{R}$ & $S U(5)^{3}$ & $S U(5)^{2} U(1)_{R}$ & $U(1)_{R}$ & $U(1)_{R}^{3}$ \\
\hline 27 & $Q$ & $\square$ & $1 / 5$ & 27 & $-108 / 5$ & -108 & $-1728 / 25$ \\
78 & $\lambda$ & 1 & 1 & 0 & 0 & 78 & $78=1950 / 25$ \\
& Total & & & 27 & $-108 / 5$ & -30 & $222 / 25$ \\
\hline \hline \multirow{3}{*}{} & $I_{1}$ & س & $3 / 5$ & 44 & $-56 / 5$ & -14 & $-56 / 25$ \\
& $I_{2}$ & 画 & $6 / 5$ & -15 & $42 / 5$ & 10 & $2 / 5$ \\
& $I_{3}$ & 田 & $9 / 5$ & 15 & $168 / 5$ & 40 & $128 / 5$ \\
& $I_{4}$ & 而 & $12 / 5$ & -44 & $196 / 5$ & 49 & $2401 / 25$ \\
& $W_{\alpha}^{2} Q^{3}$ & Ш & $13 / 5$ & 44 & $224 / 5$ & 56 & $3584 / 25$ \\
& $W_{\alpha}^{2} Q^{3}$ & 甲 & $13 / 5$ & 16 & $176 / 5$ & 64 & $4096 / 25$ \\
& $W_{\alpha}^{2} Q^{3}$ & $\overline{\boldsymbol{\theta}}$ & $13 / 5$ & -1 & 16 & 64 & $1024 / 25$ \\
& $W_{\alpha}^{2} Q^{6}$ & 口 & $16 / 5$ & 1 & $11 / 5$ & 11 & $1331 / 25$ \\
\hline
\end{tabular}

We will now make a few observations, encountered during the search for a dual. To begin, we will make the hypothesis that there does exist a free dual description. This implies that the $S U(5)$ global symmetry is explicitly realized and that $I_{1}$ is the only 
gauge singlet which is an elementary field in the dual. Thus, the dual description found for $\operatorname{Spin}(8)$ in $[46,52]$ will not be helpful here. In particular, this dual description contains several gauge-singlets transforming as 5-dimensional 4-index symmetric tensors of their global $S U(2)$. These singlets cannot all come from the free baryon $I_{1}$, but must come from $I_{2}$ or $I_{3}$. This means that their example, although an impressive accomplishment by itself, has not been "fully" dualized.

One key problem in narrowing the search for a dual for $E_{6}$ with 5 flavors is understanding how to generate the baryons $I_{2}, I_{3}, I_{4}$ without generating undesirable invariants at the same time. Another clue is the matching of the 't Hooft anomaly for $S U(5)^{3}$. The 27 coming from the electric theory is badly matched by the 44 coming from the baryon $I_{1}$. Where do the -17 come from? -17 here is a rather large number in this context. One could imagine a decomposition $17=2+3 \cdot 5=5+3 \cdot 4$. This would mean three gauge groups in the dual, with fields transforming under $G_{1} \times G_{2} \times G_{3} \times S U(5)$ as $(2,1,1, \overline{5}) \oplus(1,3,5, \overline{5})$ or as $(5,1,1, \overline{5}) \oplus(1,3,4, \overline{5})$. Such examples generate lots of unwanted invariants. To make progress, one would have to develop an understanding of how a superpotential can remove such unwanted invariants.

One simple way to avoid generating lots of unwanted invariants is to have $I_{2}, I_{3}$ as fundamental fields in the dual, but transforming under a $U(1)$ gauge symmetry. This is not necessarily an unattractive possibility, since free dual descriptions are typically not asymptotically free. However, this provides little help in cancelling the $S U(5)^{3}$ anomaly. One can wonder if the techniques of [81] could be of more general applicability and allow us to get information on what the free dual quarks could be, or at least what the rank or dimension of the dual gauge group is. Perhaps string theory constructions could also shed light on this problem.

Another curious feature is that the invariants of $S U(3)$ with 5 symmetric tensors are very similar to the invariants of $E_{6}$ with 5 fundamentals: namely, $I_{1}, I_{2}$, and $I_{3}$ are identical, while $I_{4}$ differs. The constraints among these invariants, and the chiral rings, and the glueballs, are of course different. It is not clear whether this observation has any significance.

\section{$3.2 \operatorname{Spin}(16)$ with one spinor and two vectors}

This is another example of an asymptotically free theory which is in a non-Abelian Coulomb phase at long distances, and which must have a dual description. Spin(16) has two real 128-dimensional spinor representations of opposite chiralities. Let's consider the theory with just one spinor $Q=128_{+}$, and with $N$ vectors $V=16$. There is a choice of the $R$-charge for which the $R$-charge of $Q$ and $V$ are the same, and equal to $1-\frac{7}{8+N}$. This is appropriate, since both $Q^{2}$ and $V^{2}$ are invariants. 
For $N=0$, the theory confines [16], with the spectrum of invariants $Q^{2}, Q^{8}, Q^{12}$, $Q^{14}, Q^{18}, Q^{20}, Q^{24}, Q^{30}$, as follows from knowledge of the second Casimir invariants of $E_{8}$. We do not consider the case $N=1$ here. For $N=2$, along the $\operatorname{Spin}(14)$ flat direction, $Q$ breaks into $64+64^{\prime}$. One of the 64 then Higgses $\operatorname{Spin}(14)$ to $G_{2} \times$ $G_{2}$ [16]. The remaining 64-dimensional spinor presumably decomposes into $(7,7) \oplus$ $(7,1) \oplus(1,7) \oplus(1,1)$, and these $G_{2}$ theories are known to be in a non-Abelian Coulomb phase. Since $Q^{2}$ and $V^{2}$ have $R$-charge equal to $3 / 5$, which is less than $2 / 3$, the $\operatorname{Spin}(16)$ theory with $N=2$ must have a dual description. The difficulties in finding a dual for this theory are clearly of a very different nature than for $E_{6}$ and we will have nothing further to say about it here.

\section{Invariants of $E_{6}$ with fundamentals}

Our main result in this paper is a list of the polynomially independent invariants of multiple copies of the fundamental representations of $E_{6}$ and $E_{7}$, up to degree 18. We find it convenient to express our results in the context of supersymmetric gauge theories, even though they have a more general range of application. For $E_{6}$ with chiral superfields in the 27-dimensional fundamental representation, three invariants were previously well-known, at least in the gauge theory community:

$$
I_{1}=(\mathbf{2 7})_{[3]}^{3}=\square \quad I_{2}=(\mathbf{2 7})_{\left[2^{3}\right]}^{6}=\bigoplus \quad I_{4}=(\mathbf{2 7})_{\left[3^{4}\right]}^{12}=\bigoplus
$$

However, an invariant of lower degree than $I_{4}$, of degree 9 , had attracted little attention:

$$
I_{3}=(\mathbf{2 7})_{\left[1^{5} 2^{2}\right]}^{9}=\bigoplus \text { }
$$

Previously, it had been unclear whether

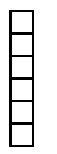

should be included as an independent invariant of $E_{6}$. We find here that $E_{6}$ does not have such an invariant. It is however an independent invariant of the symmetric tensor of $S U(3)$, as stated below in the section on $S U(3)$. We found two more invariants of degree 12 :

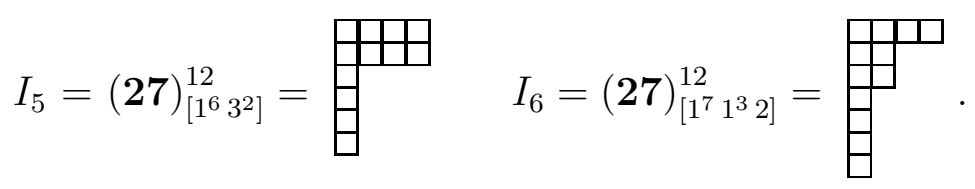


And in degree 15, we found five more invariants:

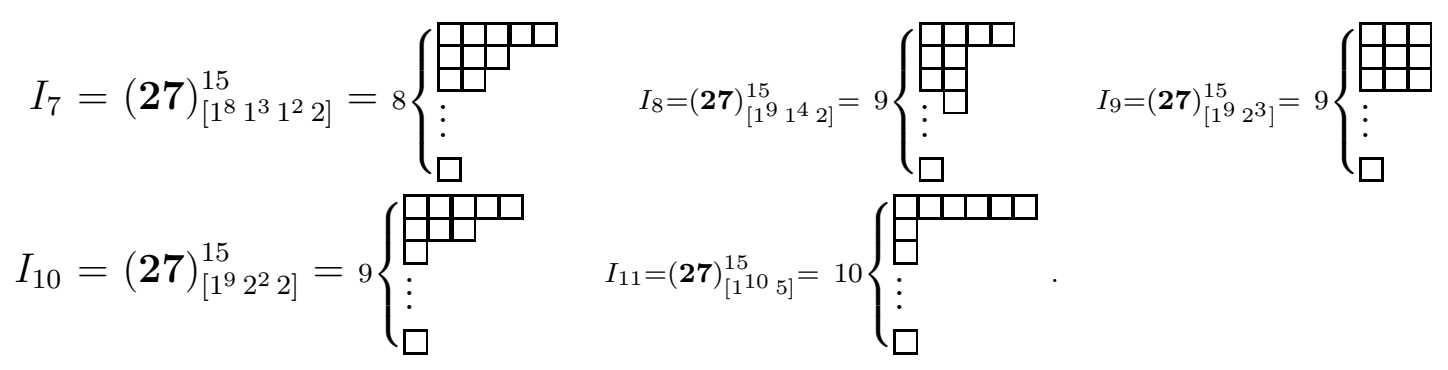

There were no constraints among the invariants up to degree 12, but five constraints (first-order syzygies) arise in degree 15:

$$
\begin{aligned}
& C_{1}^{(15)}=\left(I_{1} I_{4}+I_{2} I_{3}=0\right)_{\left[2^{5} 1^{4} 1\right]}=\bigoplus \\
& C_{2}^{(15)}=\left(I_{1} I_{2}^{2}+I_{1}^{2} I_{3}+I_{1} I_{4}+I_{2} I_{3}=0\right)_{\left[1^{5} 2^{4} 2\right]}=\bigoplus^{\square} \\
& C_{3}^{(15)}=\left(I_{2} I_{3}=0\right)_{\left[1^{6} 1^{5} 1^{4}\right]}=\bigoplus \\
& C_{4}^{(15)}=\left(I_{1}^{2} I_{3}+I_{1} I_{2}^{2}+I_{1} I_{5}+I_{2} I_{3}=0\right)_{\left[1^{6} 2^{3} 1^{2} 1\right]}=\bigoplus \\
& C_{5}^{(15)}=\left(I_{2} I_{3}+I_{1} I_{6}=0\right)_{\left[1^{7} 1^{4} 2^{2}\right]}=\bigoplus .
\end{aligned}
$$

In these expressions for the constraints, we indicate that a linear combination of invariants is constrained, along with the Young tableau describing how the indices are meant to be contracted. We did not check whether some of the coefficients in these linear combinations could be zero. And in degree 18, there are nine more invariants:

$$
\begin{aligned}
& I_{12}=(27)_{\left[1^{10} 1^{4} 1^{2} 2\right]}^{18}=10\left\{\begin{array}{l}
I_{13}=(\mathbf{2 7})_{\left[1^{10} 2^{3} 1^{2}\right]}^{18}=10 \\
\vdots \\
\square
\end{array}\right.
\end{aligned}
$$

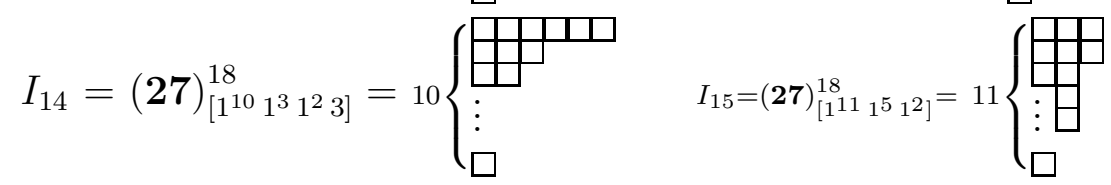

$$
\begin{aligned}
& I_{16}=(\mathbf{2 7})_{\left[1^{11} 1^{4} 1^{2} 1\right]}^{18}=11\left\{\begin{array}{l}
\prod_{17}=(\mathbf{2 7})_{\left[1_{11}^{1} 1^{3} 2^{2}\right]}^{18}=11 \\
\vdots \\
\square
\end{array}\right.
\end{aligned}
$$




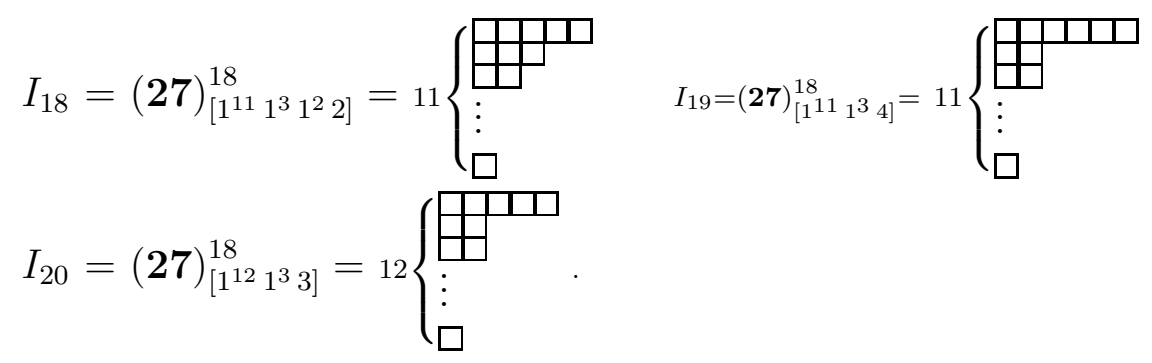

There are many constraints in degree 18, and we list them in an appendix. It is possible that this list of 20 invariants is not an exhaustive list of invariants. It would take over 50 days of computer time on a Pentium 4 machine to get the invariants of degree 21, using 1 GB of RAM, without improvement in the group theory software. We expect however that our list is almost complete. In fact, we seem to have all the invariants required for the subgroups of $E_{6}$. Along the $S O(10)$ flat direction for example, we ought to find a completely antisymmetrized baryon for the 10 -dimensional vector: $\left(\mathbf{1 0}^{10}\right)=\left[1^{10}\right]$. This invariant of $S O(10)$ must come from the invariant $I_{11}$ of $E_{6}$. As usual, information can be inferred about the invariants of the theories along the flat directions of $E_{6}$ from the knowledge of the basic invariants $I_{1}$ through $I_{20}$.

An understanding of the glueball invariants is also essential for the study of duality. This is particularly relevant for $E_{6}$ since it has $S O(N)$ subgroups, for which the glueballs play a crucial role in the matching of invariants [6]. Unfortunately, there are so many glueball invariants that we chose not to study the situation in more detail. Some results are to be found in the appendix.

\subsection{Status of the self-dual model for $E_{6}$ with 6 flavors}

In [52], Cho studied in detail the flat directions of the $E_{6}$ self-dual model of $[48,49,50]$. He considered the model along the electric $\operatorname{Spin}(8)$ flat direction, and found an invariant $d^{\prime \prime}$ of the $E_{6}$ dual theory with the quantum numbers $(\square, 5 / 2)$ under the global symmetry $S U(4) \times U(1)_{R}$. However, we point out that there are 4 invariants in the tensor product $8_{v}^{3} \otimes 8_{s} \otimes 8_{c}, 4$ invariants in $8_{s}^{3} \otimes 8_{v} \otimes 8_{c}$ and 4 invariants in $8_{c}^{3} \otimes 8_{v} \otimes 8_{s}$ all with $R$-charge $5 / 2$. There is not a scarcity of invariants that $d^{\prime \prime}$ could correspond to, although a more detailed analysis would be required to tell which of these 12 (not all independent) invariants is the right one. Thus we do not see this problem as invalidating this duality.

A potentially more serious problem is the mapping of the previously unknown invariant $I_{3}$. Corresponding to $I_{3}$, there is an invariant

$$
\tilde{\mathcal{I}}=\bar{\Phi} .
$$


However, $\tilde{\mathcal{I}}$ is not a basic invariant: it is simply the product of $\tilde{I}_{1}$ and $\tilde{I}_{2}$ from the dual theory. Furthermore, the dual superpotential renders $\tilde{I}_{1}$ redundant. Could $\tilde{\mathcal{I}}$ be considered to be a bona fide basic invariant?

Another puzzle is that the dual theory has an invariant $\tilde{I}_{3}$. This corresponds to

$$
\mathcal{I}=\text { 曺 }
$$

in the electric theory. $\mathcal{I}$ is certainly not a basic invariant, being the product of $I_{1}$ and $I_{2}$. Therefore, we need to get rid of $\tilde{I}_{3}$. Perhaps $\tilde{I}_{3}$ is made redundant by the dual superpotential. That might force us to relax the requirements of duality to rational or algebraic independence of the invariants instead of the stronger polynomial independence. Our attitude is that this self-dual model is complicated and that we do not have the technology to decisively confirm or disprove this duality ${ }^{3}$.

\section{Invariants of $E_{7}$ with fundamentals}

We repeat the search for invariants of the previous section, but this time with the 56-dimensional fundamental representation of $E_{7}$. The well-known invariants are:

$$
\begin{aligned}
& I_{1}=(\mathbf{5 6})_{\left[1^{2}\right]}^{2}=\boxminus \quad I_{2}=(\mathbf{5 6})_{[4]}^{4}=\square \\
& I_{3}=(\mathbf{5 6})_{\left[3^{2}\right]}^{6}=\boxplus \quad I_{4}=(\mathbf{5 6})_{\left[2^{3} 2\right]}^{8}=\Psi^{\square} .
\end{aligned}
$$

We then find three invariants of degree 10:

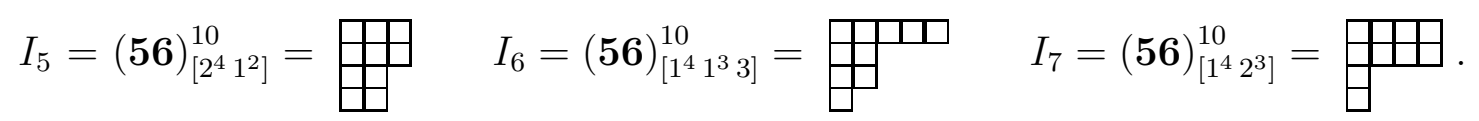

Nine invariants of degree 12 :

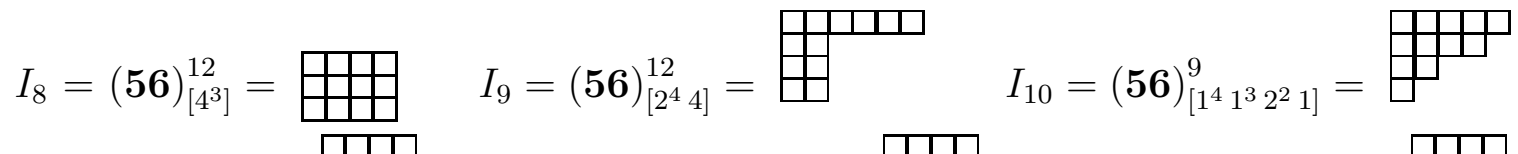

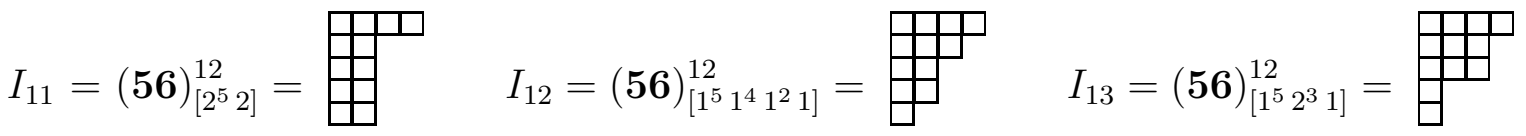

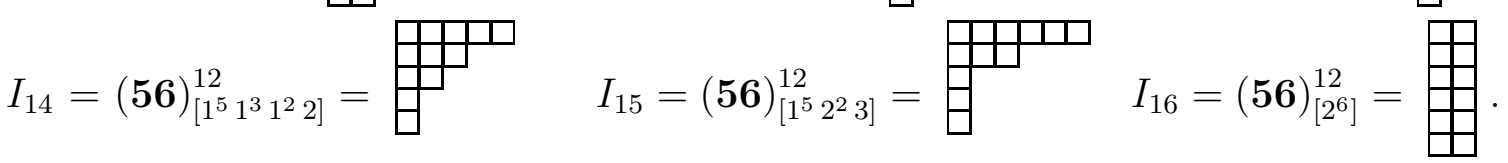

\footnotetext{
${ }^{3}$ There is another serious problem with this self-dual model [80], with the $\mathbb{Z}_{36}$ global symmetry. One way around this problem is to add the invariant $I_{2}$ to the superpotential of both electric and magnetic theories. The resulting global symmetry is $\operatorname{Spin}(6) \times U(1)_{R} \times \mathbb{Z}_{6}$. We thank Andreas Karch for reminding us.
} 
We then find thirty invariants of degree 14, where we begin to find a multiplicity of invariants with identical transformation properties. For example, the computer counted a total of 14 invariants with the Young tableau shape:

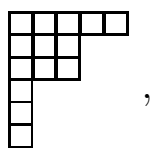

but then found that this Young tableau can be obtained in twelve different ways from the lower degree invariants ${ }^{4}$, leaving us with a count of 2 new basic invariants with that particular shape. Thus the new invariants of degree 14 are:

$$
\begin{aligned}
& (56)_{\left[3^{4} 1^{2}\right]}^{14}=\bigoplus
\end{aligned}
$$

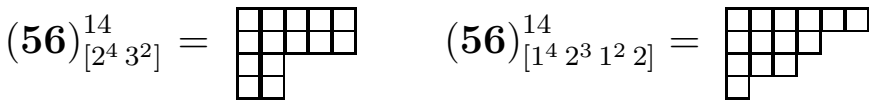

$$
\begin{aligned}
& (56)_{\left[2^{5} 1^{2} 2\right]}^{14}=\bigoplus^{\square} \\
& (56)_{\left[1^{5} 1^{4} 1^{3} 2\right]}^{14}=\bigoplus^{\square} \\
& (56)_{\left[1^{5} 2^{3} 1^{2} 1\right]}^{14}=\bigoplus \\
& (56)_{\left[1^{5} 2^{4} 1\right]}^{14}=\bigoplus \\
& (56)_{\left[1^{5} 1^{4} 2^{2} 1\right]}^{14}=\bigoplus \\
& (56)_{\left[1^{5} 1^{4} 1^{3} 1^{2}\right]}^{14}= \\
& (56)_{\left[1^{5} 1^{4} 1^{2} 3\right]}^{14}=\underbrace{10 D} \\
& (56)_{\left[1^{5} 1^{3} 2^{2} 2\right]}^{14}=\bigoplus^{-1} \\
& (56)_{\left[1^{5} 2^{3} 3\right]}^{14}=\bigoplus^{1} \\
& (56)_{\left[1^{5} 1^{3} 1^{2} 4\right]}^{14}=\bigoplus^{\bigoplus D} \\
& (56)_{\left[2^{6} 1^{2}\right]}^{14}=\bigoplus \\
& (56)_{\left[1^{6} 1^{5} 1^{2} 1\right]}^{14}=\bigoplus \\
& (56)_{\left[1^{6} 1^{5} 3\right]}^{14}=\bigoplus^{\boxplus 1 D}
\end{aligned}
$$

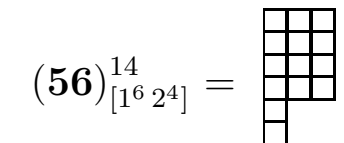

$$
\begin{aligned}
& (56)_{\left[1^{6} 1^{4} 1^{3} 1\right]}^{14}=\bigoplus \\
& 2 \times(\mathbf{5 6})_{\left[1^{6} 1^{4} 2^{2}\right]}^{14}=\bigoplus^{\boxplus \bigoplus} \\
& (56)_{\left[1^{6} 1^{4} 1^{2} 2\right]}^{14}=\bigoplus^{\bigoplus D} \\
& (56)_{\left[1^{6} 1^{4} 4\right]}^{14}=\bigoplus^{\text {बाता }} \\
& 2 \times(56)_{\left[1^{6} 2^{3} 2\right]}^{14}=\underbrace{\boxplus \amalg}
\end{aligned}
$$

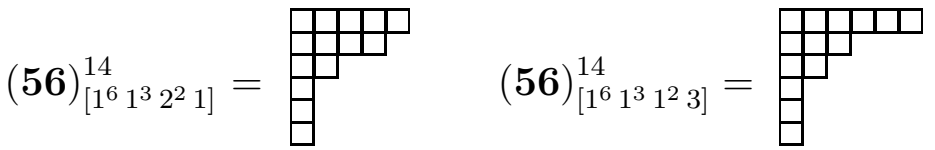

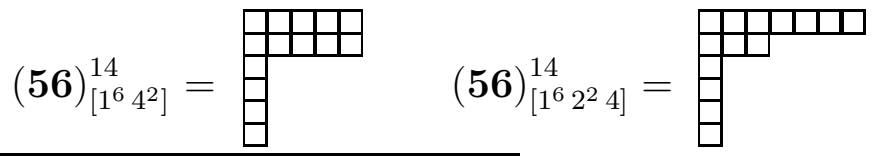

\footnotetext{
${ }^{4}$ Namely $I_{1}^{5} I_{2}, I_{1}^{4} I_{3}, I_{1} I_{3}^{2}, I_{1} I_{13}, I_{1} I_{14}, 2 \times I_{1}^{2} I_{2} I_{3}, 2 \times I_{1}^{2} I_{6}, 3 \times I_{1}^{3} I_{4}$.
} 


$$
(56)_{\left[1^{7} 1^{5} 2\right]}^{14}=\bigoplus^{\square} \text { 。 }
$$

Then we find 125 basic invariants of degree 16 (from here on, we find the Young tableau generating macro written by J. Distler for [50] extremely useful):
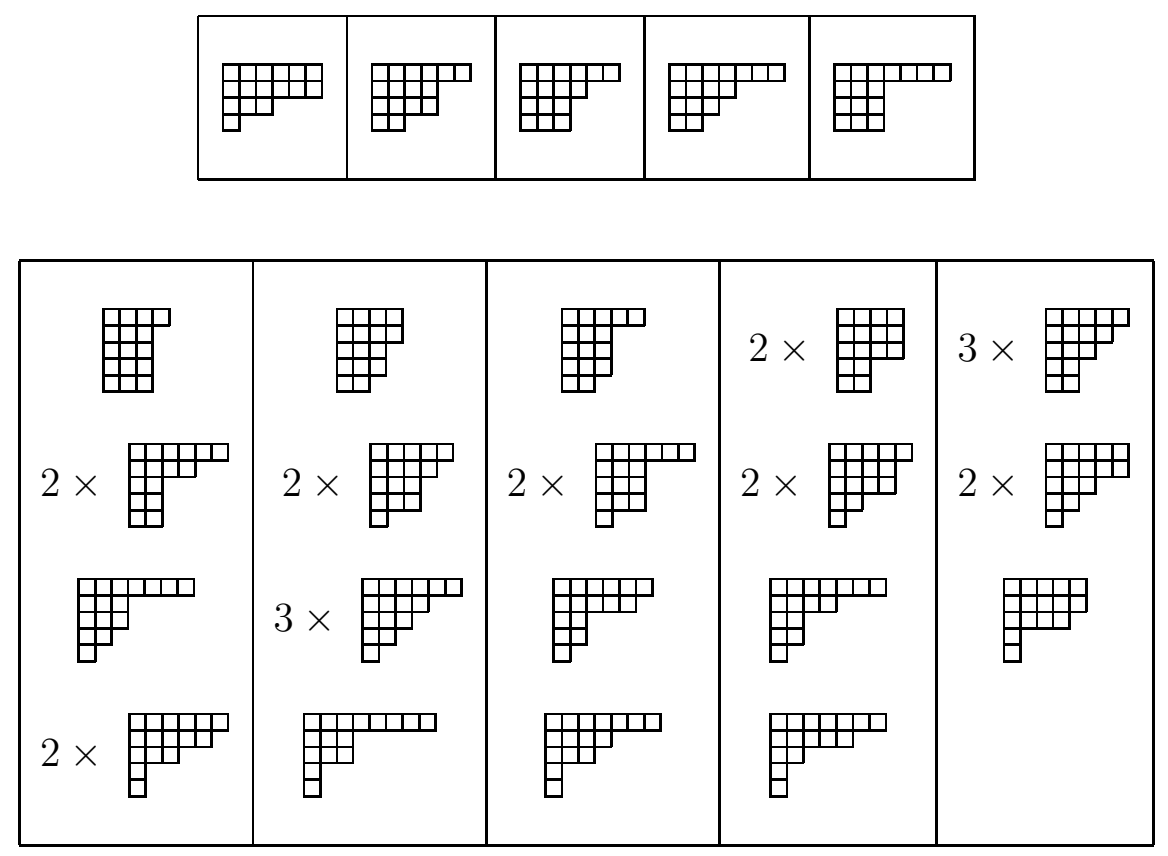

\begin{tabular}{|c|c|c|c|c|c|}
\hline 电 & 禺 & $2 \times$ 㬰 & 巴 & $2 \times$ 巴 & $2 \times$ 曲 \\
\hline $3 \times \bigoplus$ & $2 \times \bigoplus$ & 曺 & 册 & $2 \times$ 㞎 & 册 \\
\hline $4 \times \bigoplus$ & $4 \times \bigoplus$ & $2 \times$ 田 & $3 \times{ }^{\boxplus}$ & $2 \times \bigoplus$ & 曲 \\
\hline $2 \times$ 目 & $3 \times$ 册 & $2 \times$ 自 & $2 \times \bigoplus$ & & \\
\hline $2 \times{ }^{-1}$ & 四 & 讲曲 & 目 & & \\
\hline
\end{tabular}




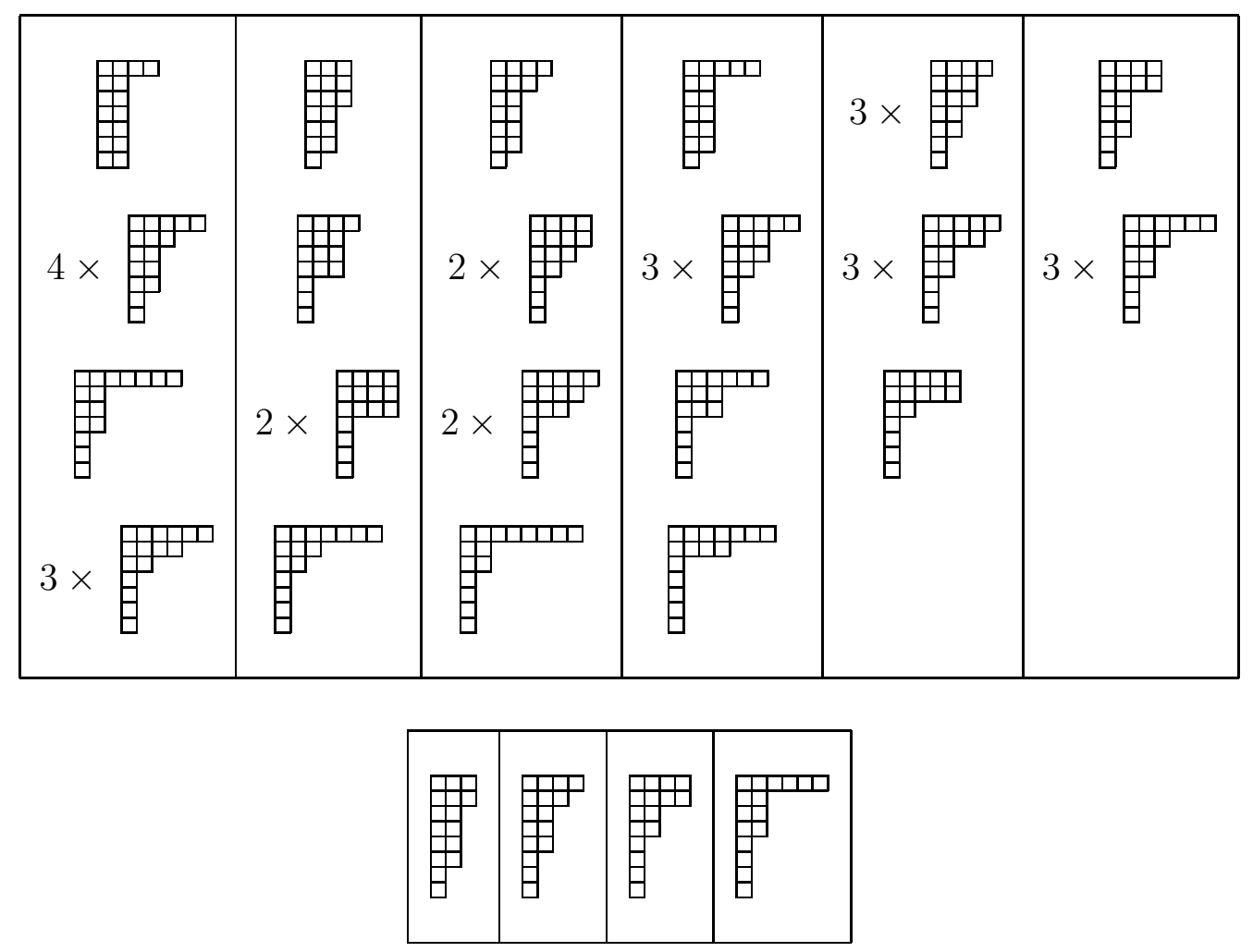

We now find 569 fundamental invariants of degree 18:

\begin{tabular}{|c|c|c|c|c|}
\hline 曲册 & 册 & $2 \times$ 曲 & 曲 & 曲 \\
\hline
\end{tabular}

\begin{tabular}{|c|c|c|c|c|c|}
\hline 巴 & $3 \times$ 曲 & $3 \times \bigoplus$ & 巴 & $6 \times \bigoplus$ & $3 \times \circledast$ \\
\hline $2 \times$ 册 & $3 \times$ 曲 & $3 \times \bigoplus^{(1}$ & $4 \times{ }^{\boxplus}$ & 曲田 & 舟 \\
\hline 曲 & $2 \times \bigoplus$ & $2 \times$ 曲 & $4 \times$ 柬 & $3 \times \bigoplus$ & $2 \times$ (10س \\
\hline $3 \times \bigoplus$ & $2 \times$ ( & 册田 & $3 \times \bigoplus$ & $2 \times{ }^{2}$ & \\
\hline$\bigoplus$ 巴业 & 册 & 巴曲 & (س & & \\
\hline
\end{tabular}




\begin{tabular}{|c|c|c|c|c|c|}
\hline 册 & 册 & $5 \times \bigoplus$ & $2 \times$ 耕 & $2 \times$ 曲 & $6 \times$ 册 \\
\hline $4 \times$ 囝 & $4 \times$ 曺 & $2 \times$ 批 & سمب & 册 & $3 \times$ 曲 \\
\hline $3 \times \bigoplus$ & $10 \times \bigoplus$ & $5 \times \bigoplus^{\square}$ & $7 \times \bigoplus$ & $6 \times \bigoplus$ & $12 \times \bigoplus$ \\
\hline $4 \times{ }^{\text {m }}$ & $4 \times$ 册 & $4 \times \bigoplus$ & سمسمب & $3 \times$ 册 & $5 \times$ 册 \\
\hline
\end{tabular}

\begin{tabular}{|c|c|c|c|c|c|}
\hline $7 \times$ 册 & $6 \times$ 曲 & $4 \times$ 进 & $5 \times$ 曲 & $8 \times \bigoplus^{\boxplus}$ & 10× \\
\hline $8 \times$ 四 & $2 \times$ سח & $4 \times$ 册 & $3 \times$ 电 & $3 \times$ سח & $2 \times$ 电册 \\
\hline 䎴 & $3 \times$ 册 & $3 \times$ 囲 & $5 \times$ 囲 & $2 \times$ 四 & \\
\hline שח & 田册 & $2 \times$ سח & ه & 电卌 & \\
\hline
\end{tabular}

\begin{tabular}{|c|c|c|c|c|c|c|}
\hline $2 \times$ & 翈 & $2 \times$ 䎴 & سمب & $2 \times$ 㞎 & $6 \times \bigoplus^{\circ}$ & 5×册 \\
\hline $5 \times$ & 册 & 4×弗 & שח & 长 & $4 \times \underset{丹}{巴}$ & $6 \times \bigoplus$ \\
\hline $4 \times$ & 册 & $13 \times \bigoplus$ & $9 \times$ 囲 & $4 \times$ 耙 & $10 \times \underset{\boxplus}{\boxplus}$ & $5 \times$ ए \\
\hline
\end{tabular}




\begin{tabular}{|c|c|c|c|c|c|}
\hline 田 & $3 \times$ 屎 & $7 \times$ 禹 & $5 \times \bigoplus^{10}$ & $7 \times$ 屎 & $8 \times \underbrace{\boxplus}$ \\
\hline $14 \times \underset{\text { 田 }}{\square}$ & $7 \times \bigoplus^{\boxplus}$ & $8 \times$ 囲 & $8 \times \oiint_{\text {日 }}^{\text {田 }}$ & $4 \times$ 䡒 & 进 \\
\hline $3 \times$ 囲 & $5 \times \sqrt{\text { 目 }}$ & $6 \times$ 囲 & $7 \times \underbrace{\text { 目 }}$ & $2 \times$ 每 & $2 \times \bigoplus^{\text {目 }}$ \\
\hline $5 \times \underbrace{\boxplus \#}_{\mathrm{E}}$ & $4 \times$ 每 & שח & $\begin{array}{l}\mathrm{PH} \\
\mathrm{B}\end{array}$ & $\begin{array}{l}\mathrm{AH} \\
\mathrm{B}\end{array}$ & 昌 \\
\hline
\end{tabular}

\begin{tabular}{|c|c|c|c|c|c|}
\hline 用 & $2 \times$ 伥 & 井 & $2 \times \underset{丹}{巴}$ & $4 \times$ 耙 & $4 \times$ 浗 \\
\hline $4 \times$ 弗 & $2 \times$ 曺 & $3 \times \underset{\text { 浗 }}{ }$ & $5 \times \underset{\Theta}{巴}$ & $9 \times$ 㞎 & $6 \times$ 册 \\
\hline $6 \times$ 四 & $\begin{array}{l}\text { मात } \\
\text { 田 }\end{array}$ & $5 \times$ 冉 & 讲 & 册 & $10 \times \underset{\text { 目 }}{\bigoplus}$ \\
\hline
\end{tabular}

\begin{tabular}{|c|c|c|c|c|c|}
\hline $6 \times \underbrace{\text { 目 }}$ & $5 \times$ 肁 & $6 \times$ 田 & $6 \times \bigoplus^{\text {目 }}$ & $\begin{array}{l}\text { 里 } \\
8\end{array}$ & $3 \times$ 昌 \\
\hline $6 \times \sqrt{\text { 昌 }}$ & 四 & $3 \times$ 䏣 & $4 \times$ 目 & & \\
\hline $2 \times$ 畐 & מسחسمت & $2 \times$ 国 & سمبت & & \\
\hline
\end{tabular}




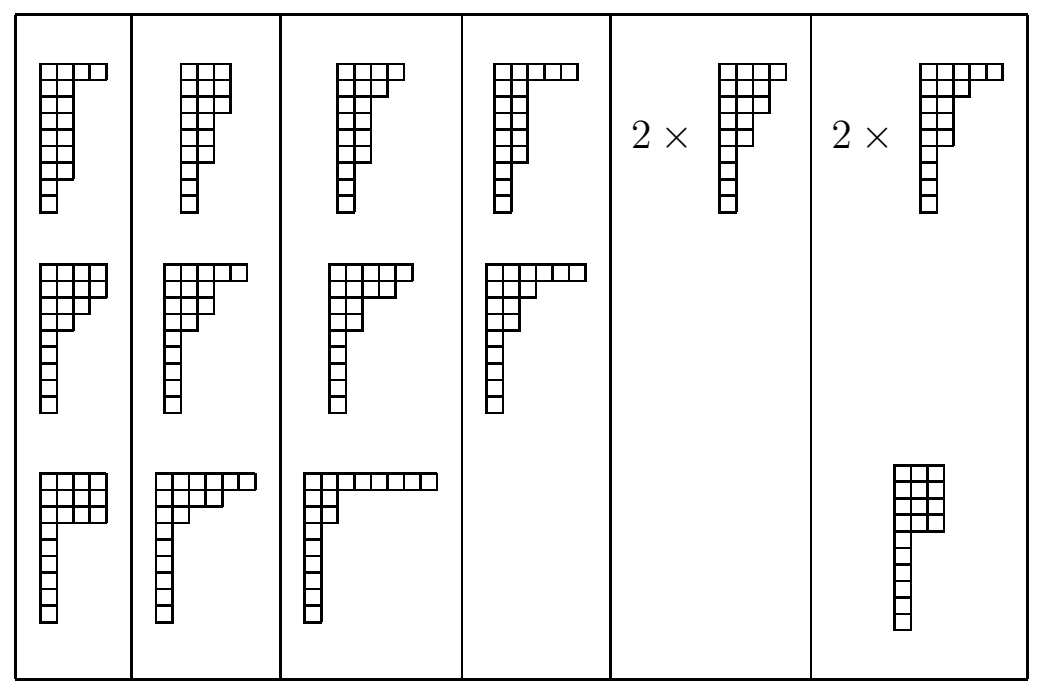

So far, we have identified 740 basic invariants, up to degree 18. Since $E_{7}$ has an $S O(12)$ subgroup, there is at least a $(\mathbf{1 2})^{12}$ invariant along this flat direction. We found only one invariant with as many as 10 antisymmetrized boxes thus far. This indicates that many more invariants are yet to be found.

\subsection{Status of the self-dual model for $E_{7}$ with 4 flavors}

In [52], Cho studied in detail the flat directions of the $E_{7}$ self-dual model of $[48,50]$ and found some problems in matching invariants. As for $E_{6}$ with 6 flavors, we find that invariants had been missed; while some of the new invariants solve the problems mentioned in [52], others cause new problems. The list of invariants for $E_{7}$ with 4 flavors have the following $S U(4) \times U(1)_{R}$ charge:

\begin{tabular}{|c|c|c|}
\hline$(\mathrm{\theta}, 1 / 2)$ & (שים, 1) & $(\boxplus, 3 / 2)$ \\
\hline (田, 2) & $(日, 5 / 2)$ & (ब⿴囗十, $5 / 2)$ \\
\hline$(\boxplus, 5 / 2)$ & $($ (册, 3) & $(\boxplus, 3)$ \\
\hline (سח (1, 3) & $(\boxplus, 7 / 2)$ & $\left(\boxplus^{\prime}, 7 / 2\right)$ \\
\hline$($ 田, 7/2) & $(日, 7 / 2)$ & $($ (丑, 4) \\
\hline$\left(\mathbb{\boxplus}^{\square}, 4\right)$ & $\left(\oplus^{\square}, 4\right)$ & $\left(\oplus^{\square}, 4\right)$ \\
\hline (שח, 4) & $(\bigoplus \#, 9 / 2)$ & $(日, 9 / 2)$ \\
\hline$($ 曲, 9/2) & (电丑, 9/2) & (9/2, سس山) \\
\hline
\end{tabular}

A few representations in this table are complex and are then potentially dangerous. A much more detailed analysis would be required to argue either way whether this duality is valid or not ${ }^{5}$.

\footnotetext{
${ }^{5}$ Just as for the $E_{6}$ self-dual model, there is a serious problem with matching the global discrete
} 


\section{Examples with Other Groups}

We have chosen a few other examples that illustrate the complexity that one encounters for some familiar rings that arise for supersymmetric gauge theories.

\subsection{The ring of $S U(2)$ with fundamentals}

In this section, we describe the simplest example of all in greater detail. We construct explicitly the free resolution of the rings, again up to degree 18 . The moduli space of $S U(2)$ with $2 N$ doublets is described by one flat direction. Alternatively, it can be described in a gauge invariant way by the invariant [2]:

$$
V_{i j}=\epsilon_{\alpha \beta} Q_{i}^{\alpha} Q_{j}^{\beta}=\boxminus \text {. }
$$

This statement is known as the "first fundamental theorem of classical invariant theory," and has of course appropriate generalizations for the $S U, S O$ and $S p$ groups. This invariant satisfies one single constraint (one first-order syzygy):

$$
Z_{1}=\operatorname{Pf} V=\text { 日 }
$$

That statement is known as the "second fundamental theorem." This constraint is itself constrained (one second-order syzygy):

$$
Z_{2}=\stackrel{\oplus}{\boxminus} \text {. }
$$

The second-order syzygy is constrained by 2 third-order syzygies:

$$
Z_{3}^{1}=\stackrel{E}{E} \quad Z_{3}^{2}=\bigoplus .
$$

The third-order syzygies are constrained by 2 fourth-order syzygies:

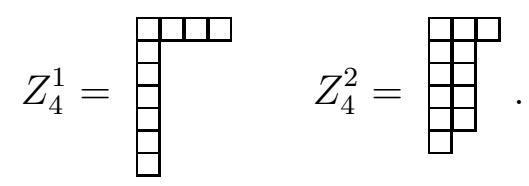

The fourth-order syzygies are constrained by 3 fifth-order syzygies:

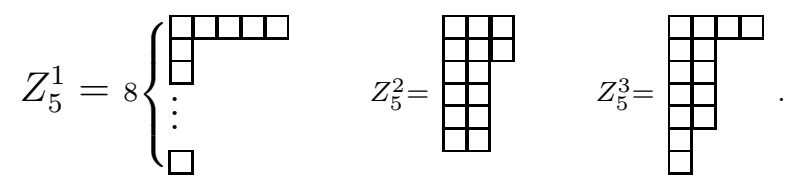

symmetry. One way around this problem is to add the invariant $I_{4}$ to the superpotential of both electric and magnetic theories. This reduces the global $S U(4)$ symmetry to $S U(3)$. 
The fifth-order syzygies are constrained by 4 sixth-order syzygies:

$$
\begin{aligned}
& z_{6}^{1}=Z_{6}^{2}=\bigoplus
\end{aligned}
$$

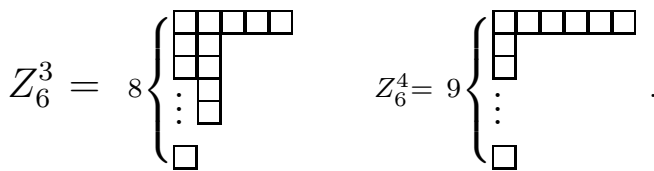

And the sixth-order syzygies are constrained by 4 seventh-order syzygies:

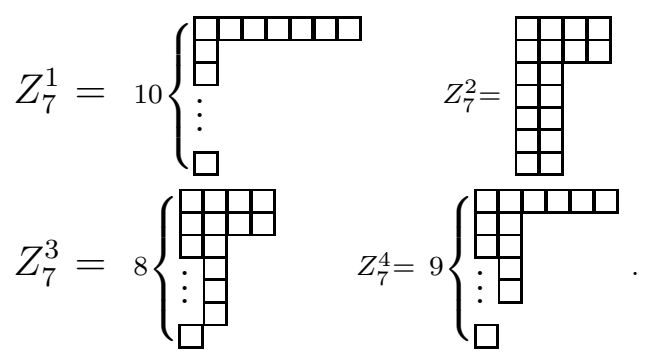

Then the seventh-order syzygies are constrained by at least one eight-order syzygies:

$$
Z_{8}=11\left\{\begin{array}{l}
\text { 政 } \\
\vdots \\
\square
\end{array},\right.
$$

and so on and so forth. Exhibiting this syzygy chain is known as constructing the free resolution of the ring. To our knowledge, the detailed form of higher-order syzygies has not played a role in physics.

\subsection{Invariants and syzygies of $G_{2}$ with fundamentals}

It is only since the eighties $[65,66]$ that the first and second fundamental theorems have been proven for $G_{2}$, and of course the other exceptional groups are well beyond reach. In this section, we will verify explicitly the results of $[65,66]$ for the invariants and the firstorder syzygies using our computer-based method. We find a complete agreement, and we extend the results to the second-order syzygies. For the 7-dimensional fundamental representation, the invariants are well-known to be:

$$
I_{1}=(\mathbf{7})_{[2]}^{2}=\square \quad I_{2}=(\mathbf{7})_{\left[1^{3}\right]}^{3}=\text { 日 } \quad I_{3}=(\mathbf{7})_{\left[1^{4}\right]}^{4}=\text { 日 } .
$$

There are first-order constraints among these invariants:

$$
C_{(6)}=\left(I_{1} I_{3}+I_{2}^{2}=0\right)_{\left[1^{5} 1\right]}=\notin \quad C_{(7)}^{1}=\left(I_{2} I_{3}=0\right)_{\left[1^{6} 1\right]}=\text { 目 }
$$




$$
\begin{aligned}
& C_{(7)}^{2}=\left(I_{1}^{2} I_{2}+I_{2} I_{3}=0\right)_{\left[1^{5} 1^{2}\right]}=\bigoplus \quad C_{(8)}^{1}=\left(I_{3}^{2}=0\right)_{\left[1^{8}\right]}=8\left\{\begin{array}{l}
\text { 是 } \\
\vdots \\
\square
\end{array}\right. \\
& C_{(8)}^{2}=\left(I_{1}^{4}+I_{3}^{2}+I_{1} I_{2}^{2}=0\right)_{\left[2^{4}\right]}=\bigoplus \quad C_{(8)}^{3}=\left(I_{1}^{2} I_{3}+\alpha I_{1} I_{2}^{2}+I_{3}^{2}=0\right)_{\left[1^{6} 1^{2}\right]}=\text { 日 } .
\end{aligned}
$$

The subscript for $C$ denotes the degree of the constraint, while the superscript just enumerates them. It is claimed in [66] that these are all the constraints, and our explicit calculation checks this to degree 16. The information in [66] is more detailed than what our computer-based method lets us achieve. For example, we see, from table I in [66], that the coefficient $\alpha$ is zero in the constraint $C_{(8)}^{3}$. Note also that our constraints are listed as (5.4.1) through (5.4.5) of table I. The other constraints in table I, as shown in [66], follow from the constraints (5.4.1) through (5.4.5), and therefore should not be included as independent constraints; our calculation explicitly confirms that. We now move on beyond the results of [66]. In degree 9, we find two second-order syzygies, i.e. constraints amongst the first-order constraints:

$$
\begin{gathered}
\left.Z_{(9)}^{1}=\left(I_{1} C_{(7)}^{1}+I_{1} C_{(7)}^{2}+I_{2} C_{(6)}=0\right)_{\left[1^{6} 1^{2} 1\right]}=\right)_{\left[1^{7} 1^{2}\right]}=Z_{(9)}=\left(I_{1} C_{(7)}^{1}+I_{2} C_{(6)} .\right.
\end{gathered}
$$

In degree 10, we find twelve second-order syzygies:

$$
\begin{gathered}
Z_{(10)}^{1}=\left(I_{1} C_{(8)}^{2}+I_{2} C_{(7)}^{2}+I_{3} C_{(6)}=0\right)_{\left[1^{5} 1^{4} 1\right]}= \\
Z_{(10)}^{2}=\left(I_{1} C_{(8)}^{3}+I_{2} C_{(7)}^{1}+I_{2} C_{(7)}^{2}+I_{1}^{2} C_{(6)}+I_{3} C_{(6)}=0\right)_{\left[1^{6} 1^{3} 1\right]} \\
Z_{(10)}^{3,4}=\left(I_{2} C_{(7)}^{1}+I_{2} C_{(7)}^{2}+I_{3} C_{(6)}=0\right)_{\left[1^{6} 1^{4}\right]}^{5}=\left(I_{2} C_{(7)}^{2}+I_{1}^{2} C_{(6)}=0\right)_{\left[1^{6} 2^{2}\right]}= \\
Z_{(10)}^{6}=\left(I_{1} C_{(8)}^{3}+I_{2} C_{(7)}^{1}+I_{2} C_{(7)}^{2}+I_{3} C_{(6)}+I_{1}^{2} C_{(6)}=0\right)_{\left[1^{7} 1^{3}\right]}
\end{gathered}
$$




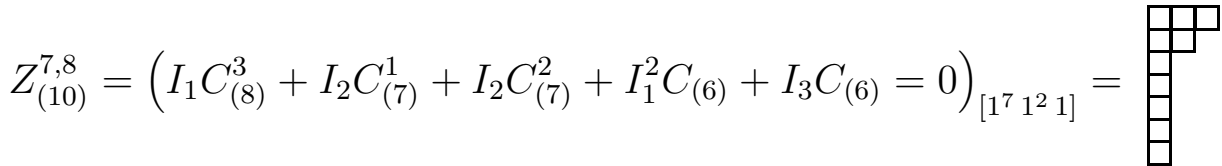

$$
\begin{aligned}
& Z_{(10)}^{9,10}=\left(I_{2} C_{(7)}^{1}+I_{2} C_{(7)}^{2}+I_{3} C_{(6)}=0\right)_{\left[1^{8} 1^{2}\right]}=8\left\{\begin{array}{l}
\boxplus \\
\vdots \\
\square
\end{array}\right. \\
& Z_{(10)}^{11}=\left(I_{1} C_{(8)}^{1}+I_{2} C_{(7)}^{1}+I_{3} C_{(6)}=0\right)_{\left[1^{8} 2\right]}=8\left\{\begin{array}{l}
{ }^{\square} \\
\vdots \\
\square
\end{array}\right. \\
& Z_{(10)}^{12}=\left(I_{1} C_{(8)}^{1}+I_{2} C_{(7)}^{1}+I_{3} C_{(6)}=0\right)_{\left[1^{9} 1\right]}=9\left\{\begin{array}{l}
\square \\
\vdots \\
\square
\end{array}\right.
\end{aligned}
$$

In degree 11, there are twenty-one second-order syzygies:

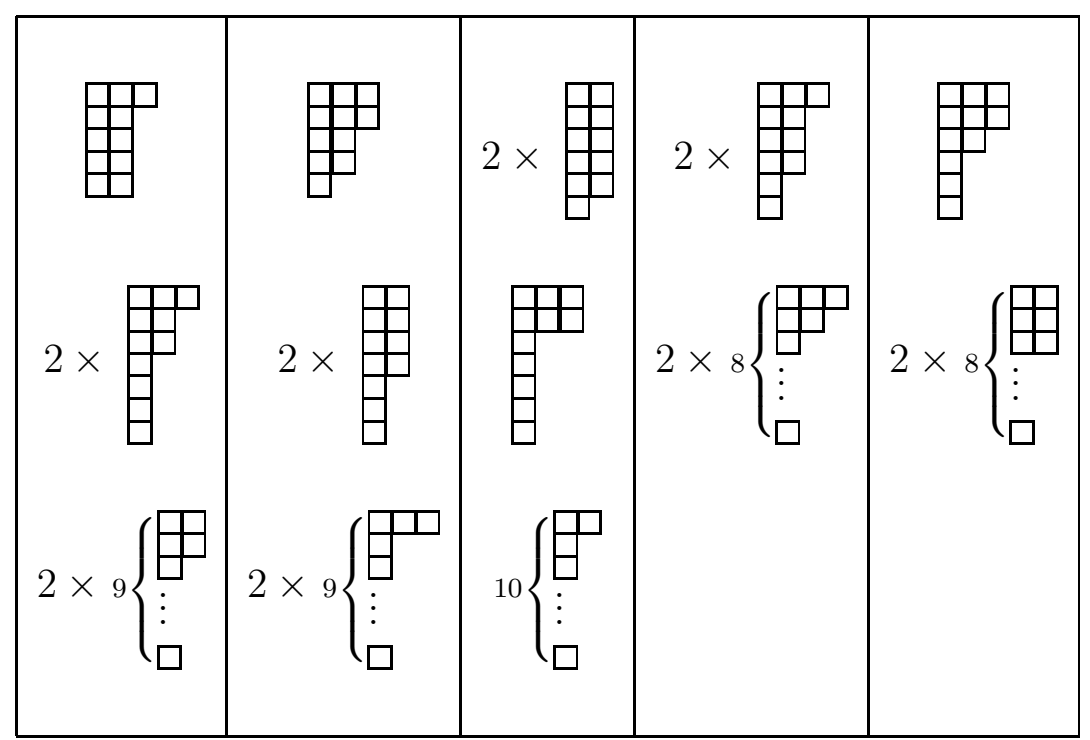

In degree 12, we find the first third-order syzygies:

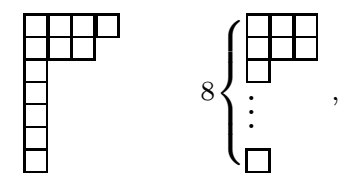

as well as sixteen more second-order syzygies: 


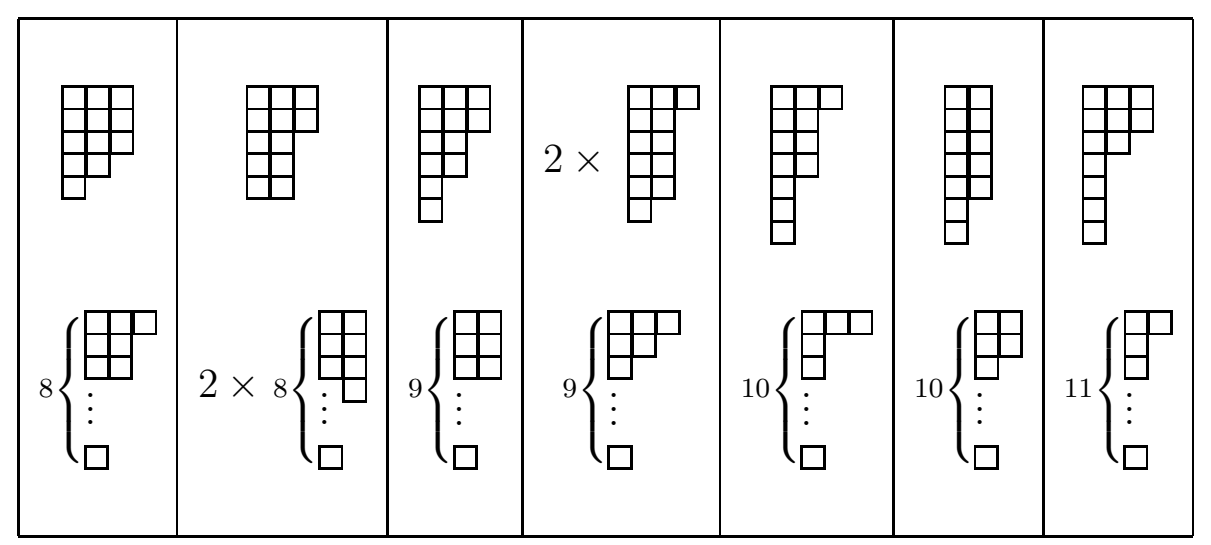

In degree 13, there is only one second-order syzygy:

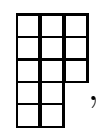

but there are many third-order syzygies:

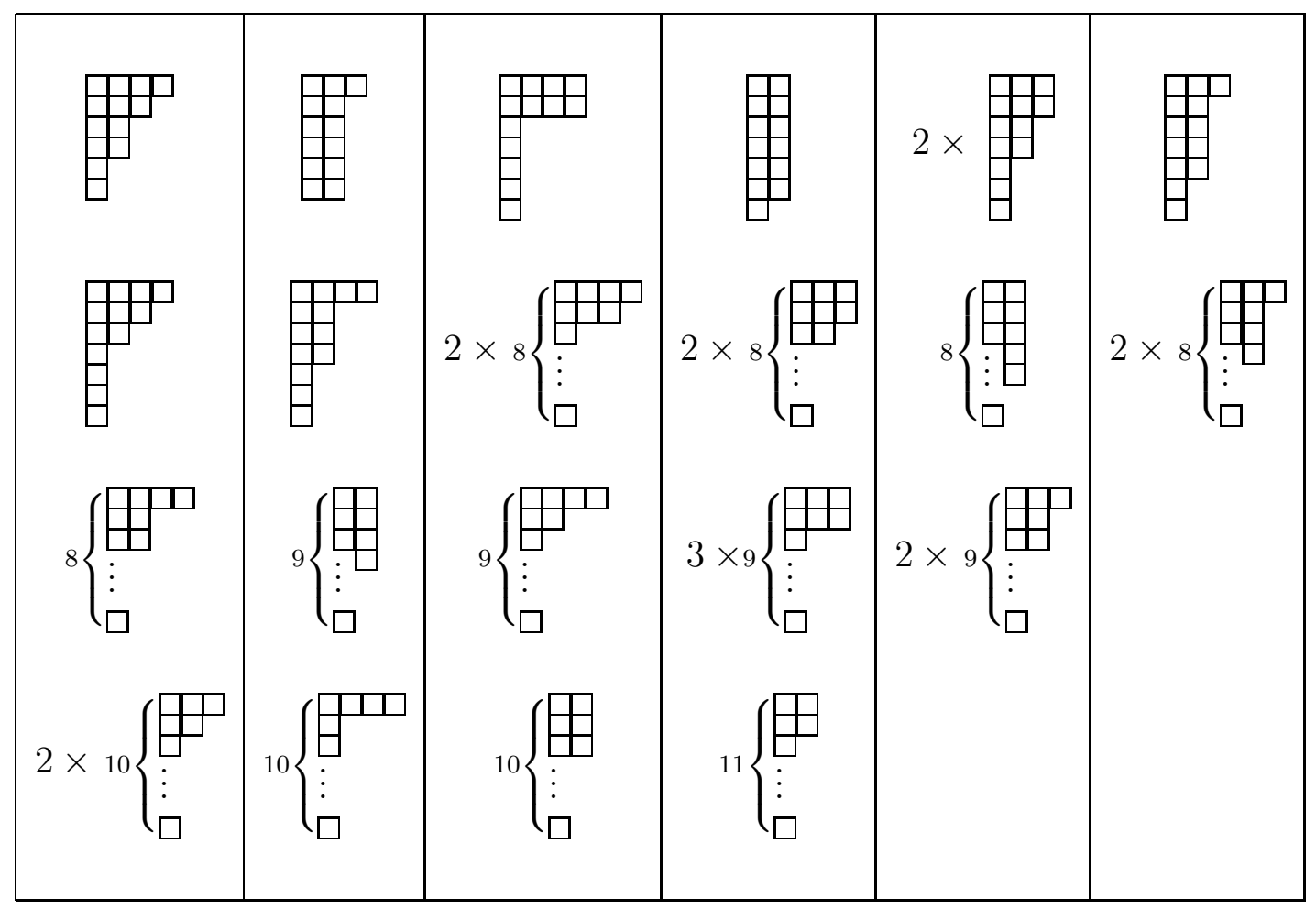

In degree 14, 15 and 16, we found no new second-order syzygy (but hundreds of new third-order syzygies). 


\subsection{Invariants of $S U(3)$ with symmetric tensors}

We repeat our search for the invariants of the 6-dimensional symmetric tensor of $S U(3)$. We find this of interest because of the close resemblance, initially, between the chiral ring of the 6 of $S U(3)$ and that of the 27 of $E_{6}$. For the six-dimensional symmetric representation, the invariants are:

$$
\begin{aligned}
& I_{1}=(\mathbf{6})_{[3]}^{3}=\square \quad I_{2}=(\mathbf{6})_{\left[2^{3}\right]}^{6}=\bigoplus \\
& I_{3}=Q_{\left[1^{6} 2^{2}\right]}^{9}=\bigoplus \quad I_{4}=(\mathbf{6})_{\left[1^{6}\right]}^{6}=\text { 日 }
\end{aligned}
$$

These appears to be all the invariants (at least up to degree 18). There is one constraint in degree 9:

$$
C_{(9)}=\left(I_{1} I_{3}=0\right)_{\left[1^{7} 2\right]}=\stackrel{\theta}{\mathrm{H}} .
$$

In degree 12, we find seventeen of constraints:

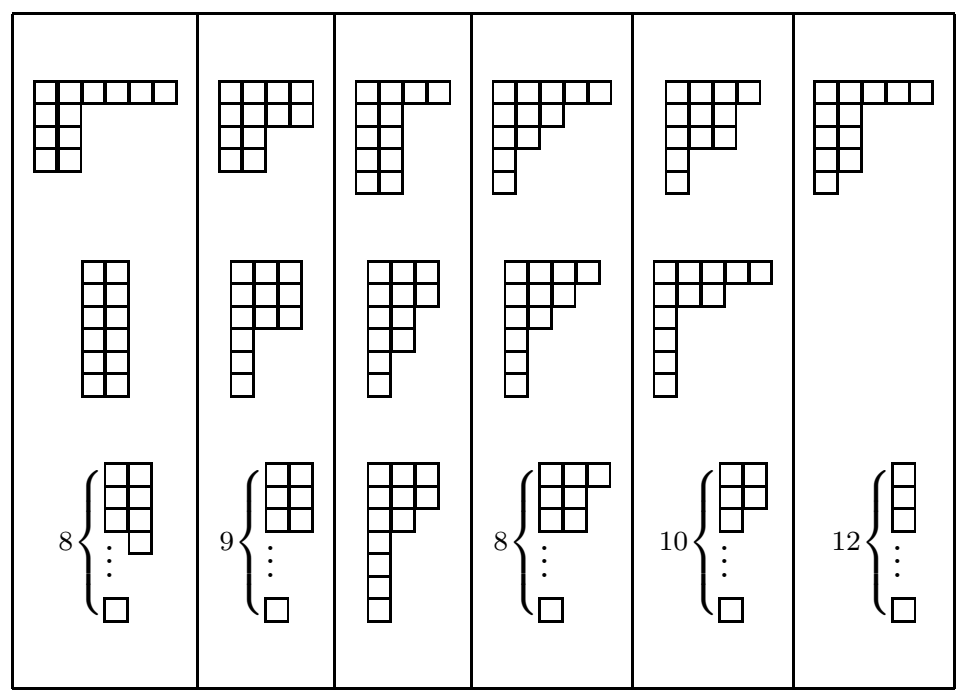

As well as degree 12 second-order syzygies:

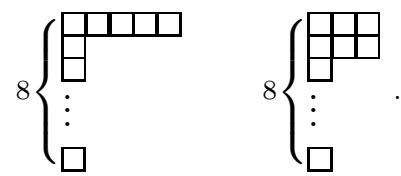




\subsection{Invariants of $S U(4)$ with symmetric tensors}

For $S U(4)$ with a 10-dimensional symmetric tensor, there is one invariant of degree 4 and three of degree 8 :

$$
\begin{aligned}
& I_{1}=(\mathbf{1 0})_{[4]}^{4}=\square \quad I_{2}=(\mathbf{1 0})_{\left[2^{4}\right]}^{8}=\bigoplus \\
& I_{3}=(\mathbf{1 0})_{\left[1^{6} 2\right]}^{8}=\underbrace{\boxminus 口} \quad I_{4}=(\mathbf{1 0})_{\left[2^{3} 2\right]}^{8}=\Psi^{\square} .
\end{aligned}
$$

We then find a large number of invariants of degrees 12 :

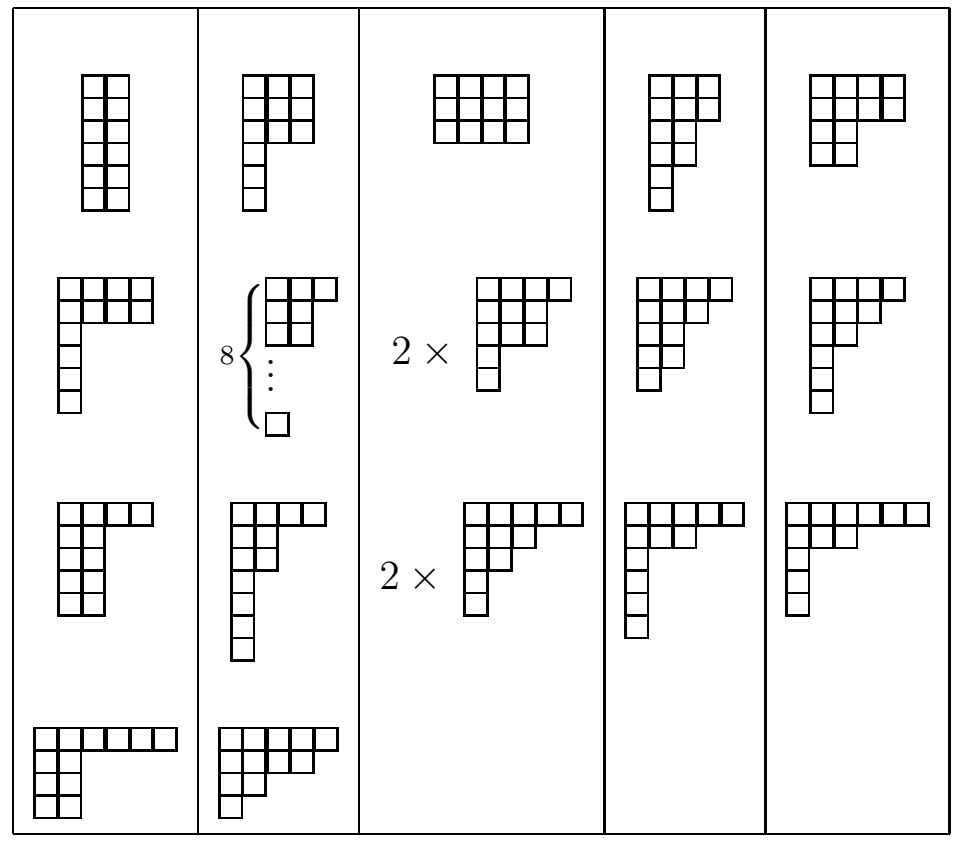

As well as one constraint of degree 12:

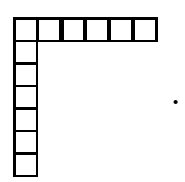

There is an impressionistic resemblance between the chiral ring of symmetric tensors of $S U(4)$ and the chiral ring of $E_{7}$ with fundamentals, at least for the invariants of degrees 4,8 and 12 . 


\subsection{Invariants of $S U 5)$ with symmetric tensors}

For the 15-dimensional symmetric tensor representation of $S U(5)$, the first few invariants are:

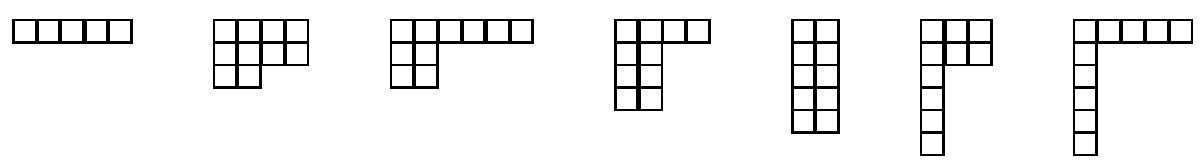

Then in degree 15, there is roughly 178 new invariants and one constraint.

\section{Conclusion}

In this paper, we have explored the structure of the chiral rings of several classes of gauge theories. We were surprised that the invariants turned out to be so complicated. This makes the search for more duals at least arduous, if not futile.

There might be a guide for finding more gauge theories that have a chance of having a simple enough solution: one can systematically calculate the homological dimension of rings of gauge theories and look for examples, (e.g. by truncating the chiral ring [23]), which have a small homological dimension.

\section{Acknowledgments}

We would like to thank Dan Freed for explanations putting classical invariant theory in context. This work was supported by NSF grant PHY-0071512. 
A. The constraints of degree 18 for $E_{6}$

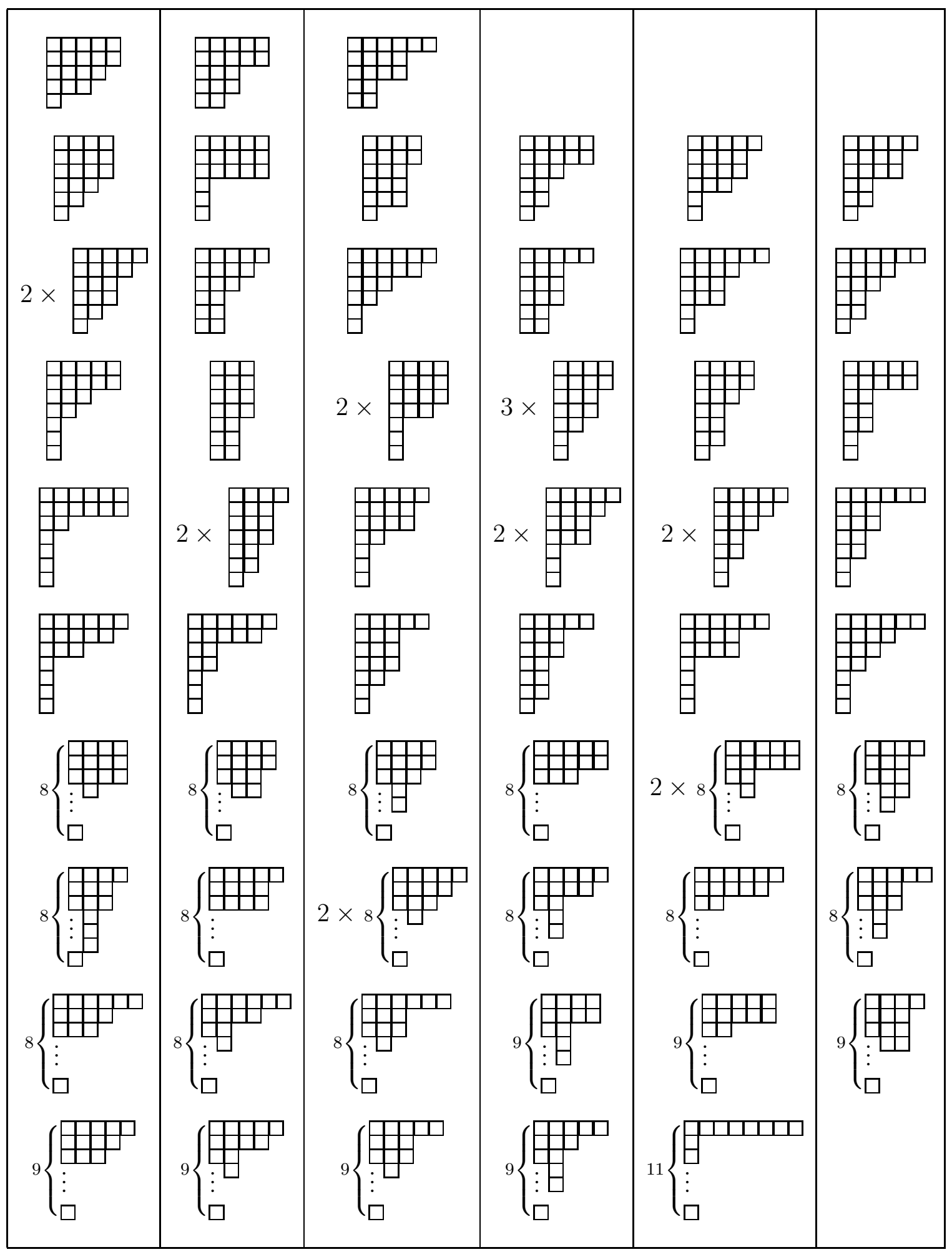




\section{B. Some glueballs of $E_{6}$}

In general, we do not understand how glueballs are mapped. For example, in the basic example of $S U\left(N_{c}\right) \leftrightarrow S U\left(N_{f}-N_{c}\right)$ of [1], the spinorial glueball superfields do not appear to have partners in the dual theory ${ }^{6}$ :

$$
W_{\alpha} Q^{N_{c}}=N_{c}-1\left\{\begin{array}{l}
\square \\
\vdots \\
\square
\end{array},\right.
$$

in the electric theory, clearly transforms differently from:

$$
\tilde{W}_{\alpha} q^{N_{f}-N_{c}}=N_{f-N_{c}-1}\left\{\begin{array}{l}
\bar{\nabla} \\
\vdots \\
\square
\end{array}=N_{c}-1\left\{\begin{array}{l}
\Phi \\
\square \\
\vdots \\
\square
\end{array}\right\} N_{f}-1\right.
$$

in the magnetic theory.

For $E_{6}$, the situation is of course much worse. We find that there is a rather large number of glueballs for $E_{6}$, the invariants involving the 78-dimensional adjoint representation for the glueball superfield $W_{\alpha}$. First, the Lorentz-spinor invariants of the form $W_{\alpha} Q^{3 n}$ :

$$
\begin{aligned}
& J_{1} \quad=(\mathbf{7 8})(\mathbf{2 7})_{\left[1^{2} 1\right]}^{3}=\square \quad J_{2}=(\mathbf{7 8})(\mathbf{2 7})_{\left[1^{3} 1^{2} 1\right]}^{6}=\bigoplus \\
& J_{3} \quad=(78)(27)_{\left[1^{4} 1^{2}\right]}^{6}=\bigoplus \quad J_{4}=(78)(27)_{\left[1^{4} 2^{2} 1\right]}^{9}=\bigoplus \\
& J_{5}=(\mathbf{7 8})(\mathbf{2 7})_{\left[1^{4} 1^{3} 1^{2}\right]}^{9}=\bigoplus \quad J_{6}=(\mathbf{7 8})(\mathbf{2 7})_{\left[1^{5} 2^{2}\right]}^{9}=\bigoplus
\end{aligned}
$$

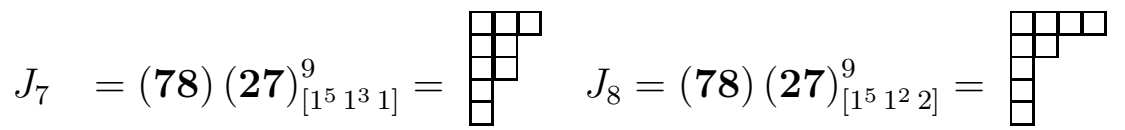

$$
\begin{aligned}
& J_{9}=(\mathbf{7 8})(\mathbf{2 7})_{\left[1^{6} 1^{2} 1\right]}^{9}=\stackrel{\Theta}{\mathrm{E}}
\end{aligned}
$$

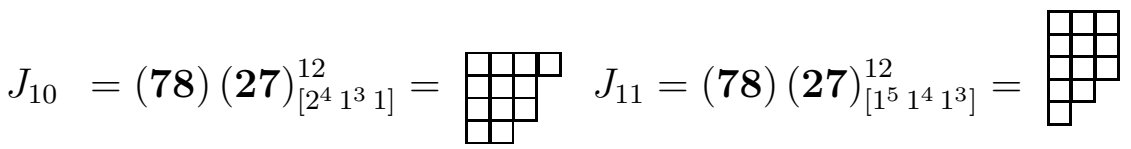

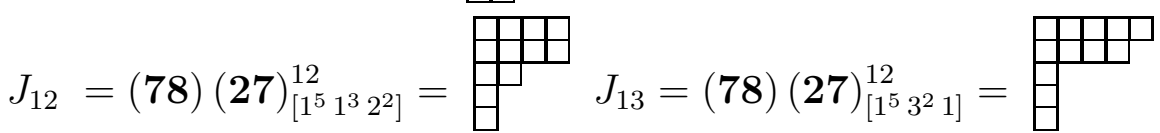

\footnotetext{
${ }^{6}$ This is actually not a problem because these invariants, although chiral, are not primary: they are descendants [82]. We will not try to discriminate between primaries and descendants in this appendix. We thank Andreas Karch and Micha Berkooz for this comment.
} 


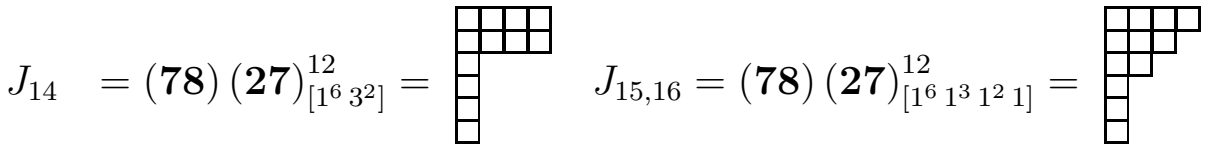

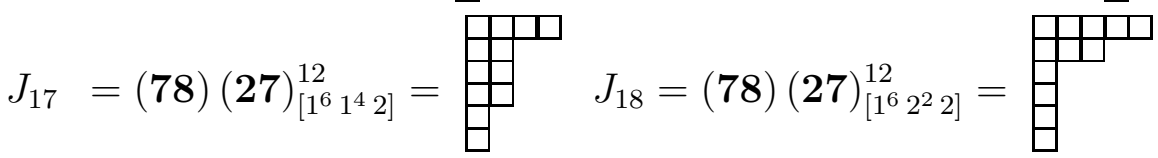

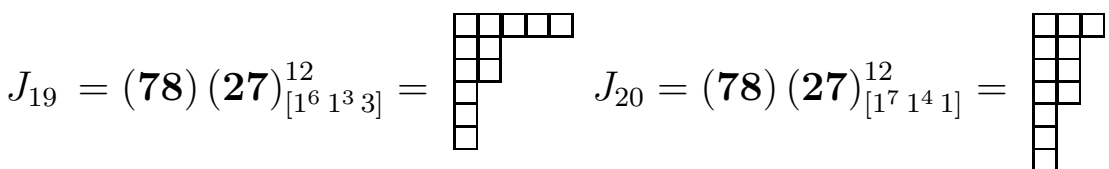

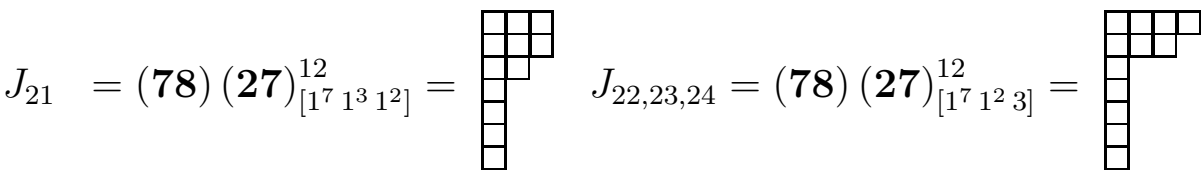

$$
\begin{aligned}
& J_{25,26}=(\mathbf{7 8})(\mathbf{2 7})_{\left[1^{7} 1^{3} 2\right]}^{12}=\bigoplus \quad J_{27}=(\mathbf{7 8})(\mathbf{2 7})_{\left[1^{8} 1^{3} 1\right]}^{12}=8\left\{\begin{array}{l}
\Phi \\
\vdots \\
\square
\end{array}\right.
\end{aligned}
$$

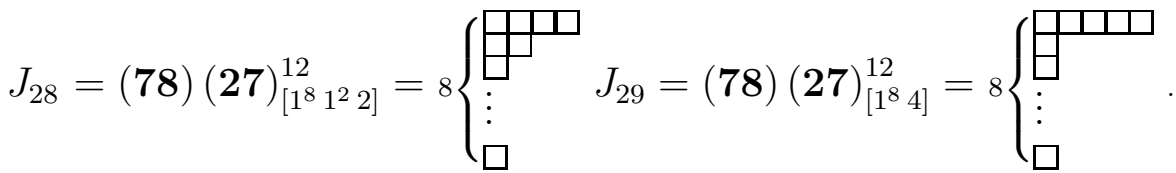

Then the Lorentz-scalar invariants of the form $W_{\alpha}^{2} Q^{3 n}$ :

$$
\begin{aligned}
& K_{1}=(\mathbf{7 8})^{2}(\mathbf{2 7})_{[3]}^{3}=\square \quad K_{2}=(\mathbf{7 8})^{2}(\mathbf{2 7})_{\left[1^{2} 1\right]}^{3}=\square \\
& K_{3}=(\mathbf{7 8})^{2}(\mathbf{2 7})_{\left[1^{2} 1\right]}^{3}=\boxplus \quad K_{4}=(\mathbf{7 8})^{2}(\mathbf{2 7})_{\left[1^{3}\right]}^{3}=\text { 日 } \\
& K_{5,6}=(\mathbf{7 8})^{2}(\mathbf{2 7})_{\left[2^{3}\right]}^{6}=\boxplus \quad K_{7,8}=(\mathbf{7 8})^{2}(\mathbf{2 7})_{\left[3^{2}\right]}^{6}=\boxplus \\
& K_{9}=(\mathbf{7 8})^{2}(\mathbf{2 7})_{\left[2^{2} 2\right]}^{6}=\boxplus \square \quad K_{10,11,12,13}=(\mathbf{7 8})^{2}(\mathbf{2 7})_{\left[1^{4} 1^{2}\right]}^{6}=\bigoplus \\
& K_{14,15,16,17,18}=(\mathbf{7 8})^{2}(\mathbf{2 7})_{\left[1^{3} 1^{2} 1\right]}^{6}=\bigoplus \quad K_{19,20}=(\mathbf{7 8})^{2}(\mathbf{2 7})_{\left[1^{1} 2\right]}^{6}=\text { 田 } \\
& K_{21}=(\mathbf{7 8})^{2}(\mathbf{2 7})_{\left[1^{5} 1\right]}^{6}=\text { 日 } \quad K_{22,23}=(\mathbf{7 8})^{2}(\mathbf{2 7})_{\left[1^{3} 3\right]}^{6}=\text { 日十 }
\end{aligned}
$$

Due to this proliferation of glueball invariants, we stop the list here. 


\section{Computer Program Code for $\mathrm{LiE}$}

The group theory program LiE can be obtained for free from a variety of sources, e.g. http://wwwmathlabo.univ-poitiers.fr/ ${ }^{\sim m a a v l / L i E / ~ . ~ I t ~ i s ~ w r i t t e n ~ i n ~} C$, easy to install and easy to use. It comes with a well-written and useful manual [83]. (There is another well-known group theory software, Schur. However it is not free.) We include some commented code below.

0 \#Write to a default file named 'monfile'

1 on monitor

2 maxobjects 2000000

3 maxnodes 12000

4 thegroup = E6; therank $=6$;

$5 \mathrm{\# n}$ is the degree of the invariants we are computing

$6 \mathrm{n}=9$;

7 setdefault Lie_group $(1, \mathrm{n})$;

8 fund $=[1,0,0,0,0,0,0,0,0]$;

$9 \mathrm{y}=$ poly_null $(\mathrm{n})$

$10 \mathrm{i} i=0$;

11 \#The next line iterates over all the partitions of $n=6$

12 for lambda row partitions(n) do

13 \#Compute the plethysm of degree $n$ of $E_{6}$

$14 \mathrm{~b}=$ plethysm (lambda, $[1,0,0,0,0,0]$, thegroup);

15 \#Compute the $S U(n)$ representation for the partition $\lambda$

$16 \mathrm{x}=$ plethysm (lambda, fund);

\# Line 16 is very slow. More efficient is to use the built-in function from_part

\# (after adding a zero to each partition to get representations of $A_{n}$ instead of $A_{n-1}$ ).

17 \#Print useful information at each iteration

$18 i i=i i+1$;

19 print("partition number:"); print(ii);

20 print("partition:"); print(x); print(lambda);

21 print("number of objects used:"); print(used);

22 print("the plethysm:");

23 print (b);

24 print("Multiplying by W_alpha:");

25 \#Tensor the plethysm $b$ with the adjoint

26 \#The coefficient $1 \mathrm{X}$ is for compatibility with $b$ because $b$ is a polynomial 
$27 \mathrm{cc}=$ tensor $(1 \mathrm{X}[0,1,0,0,0,0], \mathrm{b}$, thegroup);

28 print (cc);

29 \#Pick the singlet(s) from the final expression

$30 \mathrm{a}=\mathrm{cc}[1]$;

31 \#Check that $a$ is a singlet; if so, add it to $y$

32 if $(a==(\operatorname{coef}(\mathrm{a}, 1) *$ poly_one $($ therank $)))$ then

$33 \mathrm{y}=\mathrm{y}+(\operatorname{coef}(\mathrm{a}, 1) * \mathrm{x})$;

34 print("This is the coefficient:");

35 print $(\operatorname{coef}(\mathrm{a}, 1) * \mathrm{x})$;

36 print("This is 'y': "); print(y);

37 \# ' $y$ ' is the full list of invariants

38 \#Closes the if

$39 \mathrm{fi}$;

40 \#Activate the garbage collection:

41 \#Essential memory management!

42 gcol;

43 \#Close the do loop

44 od;

45 \#Define the previously found lower degree invariants

$46 j 1=\operatorname{plethysm}([2,1]$, fund $)$;

$47 j 2=\operatorname{plethysm}([3,2,1]$, fund $) ;$

$48 j 3=\operatorname{plethysm}([2,2,1,1]$, fund $)$;

49

$50 \mathrm{r1}=\operatorname{plethysm}([3]$, fund $)$;

$51 \mathrm{r} 2=\operatorname{plethysm}([2,2,2]$, fund $)$;

$52 r 3=\operatorname{plethysm}([3,3,1,1,1,1]$, fund $)$;

$53 \mathrm{r4}=\operatorname{plethysm}([3,3,3,3]$, fund $)$;

$54 \mathrm{r} 5=\operatorname{plethysm}([4,4,1,1,1,1]$, fund $)$;

$55 \mathrm{r} 6=\operatorname{plethysm}([4,2,2,1,1,1,1]$, fund $)$;

56 \#Compute the product of lower degree invariants among themselves

$57 f=$ tensor $(r 1, j 2+j 3)+$ tensor $\left(\operatorname{sym} \_\right.$tensor $\left.(2, r 1), j 1\right)+$ tensor $(r 2, j 1)$;

58 print("This is $f:$ ");

59 print (f);

60 print("These are the new fundamental invariants: ");

61 print $(\mathrm{y}-\mathrm{f})$; 


\section{References}

[1] N. Seiberg, "Electric - magnetic duality in supersymmetric nonAbelian gauge theories," Nucl. Phys. B 435, 129 (1995) [hep-th/9411149].

[2] N. Seiberg, "Exact results on the space of vacua of four-dimensional SUSY gauge theories," Phys. Rev. D 49, 6857 (1994) [hep-th/9402044].

[3] K. Intriligator, R. G. Leigh and N. Seiberg, "Exact superpotentials in four-dimensions," Phys. Rev. D 50, 1092 (1994) [hep-th/9403198].

[4] K. Intriligator, "'Integrating in' and exact superpotentials in 4-d," Phys. Lett. B 336, 409 (1994) [hep-th/9407106].

[5] K. Intriligator, N. Seiberg and S. H. Shenker, "Proposal for a simple model of dynamical SUSY breaking," Phys. Lett. B 342, 152 (1995) [hep-ph/9410203].

[6] K. Intriligator and N. Seiberg, "Duality, monopoles, dyons, confinement and oblique confinement in supersymmetric SO(N(c)) gauge theories," Nucl. Phys. B 444, 125 (1995) [hep-th/9503179].

[7] K. Intriligator and P. Pouliot, "Exact superpotentials, quantum vacua and duality in supersymmetric SP(N(c)) gauge theories," Phys. Lett. B 353, 471 (1995) [hep-th/9505006].

[8] S. B. Giddings and J. M. Pierre, "Some exact results in supersymmetric theories based on exceptional groups," Phys. Rev. D 52, 6065 (1995) [hep-th/9506196].

[9] I. Pesando, "Exact results for the supersymmetric G(2) gauge theories," Mod. Phys. Lett. A 10, 1871 (1995) [hep-th/9506139].

[10] P. Cho and P. Kraus, "Symplectic SUSY gauge theories with antisymmetric matter," Phys. Rev. D 54, 7640 (1996) [hep-th/9607200].

[11] C. Csaki, W. Skiba and M. Schmaltz, "Exact results and duality for Sp(2N) SUSY gauge theories with an antisymmetric tensor," Nucl. Phys. B 487, 128 (1997) [hep-th/9607210].

[12] C. Csaki, M. Schmaltz and W. Skiba, "Systematic approach to confinement in N = 1 supersymmetric gauge theories," Phys. Rev. Lett. 78, 799 (1997) [hep-th/9610139].

[13] C. Csaki, M. Schmaltz, W. Skiba and J. Terning, "Self-dual N = 1 SUSY gauge theories," Phys. Rev. D 56, 1228 (1997) [hep-th/9701191].

[14] B. Grinstein and D. R. Nolte, "Systematic study of theories with quantum modified moduli. II," Phys. Rev. D 58, 045012 (1998) [hep-th/9803139].

[15] G. Dotti, A. V. Manohar and W. Skiba, "Supersymmetric gauge theories with a free algebra of invariants," Nucl. Phys. B 531, 507 (1998) [hep-th/9803087]. 
[16] G. Dotti and A. V. Manohar, "Supersymmetric gauge theories with an affine quantum moduli space," Phys. Rev. Lett. 80, 2758 (1998) [hep-th/9712010].

[17] C. Csaki and H. Murayama, "New confining $N=1$ supersymmetric gauge theories," Phys. Rev. D 59, 065001 (1999) [hep-th/9810014].

[18] P. Cho, "Exact results in SO(11) SUSY gauge theories with spinor and vector matter," Phys. Lett. B 400, 101 (1997) [hep-th/9701020].

[19] N. Maru, "Confining phase in SUSY SO(12) gauge theory with one spinor and several vectors," Mod. Phys. Lett. A 13, 1361 (1998) [hep-th/9801187].

[20] C. Csaki and W. Skiba, "Classification of the N = 1 Seiberg-Witten theories," Phys. Rev. D 58, 045008 (1998) [hep-th/9801173].

[21] M. Klein, "More confining N = 1 SUSY gauge theories from non-Abelian duality," Nucl. Phys. B 553, 155 (1999) [hep-th/9812155].

[22] M. Klein, "Confining N = 1 SUSY gauge theories from Seiberg duality," hep-th/9904210.

[23] D. Kutasov, "A Comment on duality in $\mathrm{N}=1$ supersymmetric nonAbelian gauge theories," Phys. Lett. B 351, 230 (1995) [hep-th/9503086].

[24] D. Kutasov and A. Schwimmer, "On duality in supersymmetric Yang-Mills theory," Phys. Lett. B 354, 315 (1995) [hep-th/9505004].

[25] K. Intriligator, "New RG fixed points and duality in supersymmetric $\mathrm{SP}(\mathrm{N}(\mathrm{c}))$ and SO(N(c)) gauge theories," Nucl. Phys. B 448, 187 (1995) [hep-th/9505051].

[26] K. Intriligator, R. G. Leigh and M. J. Strassler, "New examples of duality in chiral and nonchiral supersymmetric gauge theories," Nucl. Phys. B 456, 567 (1995) [hepth/9506148].

[27] J. H. Brodie, "Duality in supersymmetric SU(N/c) gauge theory with two adjoint chiral superfields," Nucl. Phys. B 478, 123 (1996) [hep-th/9605232].

[28] M. Berkooz, "The Dual of supersymmetric SU(2k) with an antisymmetric tensor and composite dualities," Nucl. Phys. B 452, 513 (1995) [hep-th/9505067].

[29] E. Poppitz, Y. Shadmi and S. P. Trivedi, "Duality and Exact Results in Product Group Theories," Nucl. Phys. B 480, 125 (1996) [hep-th/9605113].

[30] E. Poppitz, Y. Shadmi and S. P. Trivedi, "Supersymmetry breaking and duality in SU(N) x SU(N-M) theories," Phys. Lett. B 388, 561 (1996) [hep-th/9606184].

[31] P. Pouliot, "Duality in SUSY $S U(N)$ with an Antisymmetric Tensor," Phys. Lett. B 367, 151 (1996) [hep-th/9510148]. 
[32] A. Giveon, O. Pelc and E. Rabinovici, "The Coulomb phase in N = 1 gauge theories with an LG-type superpotential," Nucl. Phys. B 499, 100 (1997) [hep-th/9701045].

[33] J. Lykken, E. Poppitz and S. P. Trivedi, "M(ore) on chiral gauge theories from D-branes," Nucl. Phys. B 520, 51 (1998) [hep-th/9712193].

[34] M. A. Luty, M. Schmaltz and J. Terning, "A Sequence of Duals for $\operatorname{Sp}(2 \mathrm{~N})$ Supersymmetric Gauge Theories with Adjoint Matter," Phys. Rev. D 54, 7815 (1996) [hepth/9603034].

[35] J. Lykken, E. Poppitz and S. P. Trivedi, "Chiral gauge theories from D-branes," Phys. Lett. B 416, 286 (1998) [hep-th/9708134].

[36] J. H. Brodie and M. J. Strassler, "Patterns of duality in N $=1$ SUSY gauge theories or: Seating preferences of theater-going non-Abelian dualities," Nucl. Phys. B 524, 224 (1998) [hep-th/9611197].

[37] R. G. Leigh and M. J. Strassler, "Accidental symmetries and N = 1 duality in supersymmetric gauge theory," Nucl. Phys. B 496, 132 (1997) [hep-th/9611020].

[38] R. G. Leigh and M. J. Strassler, "Duality of $\mathrm{Sp}(2 \mathrm{~N}(\mathrm{c}))$ and $\mathrm{S} 0(\mathrm{~N}(\mathrm{c}))$ supersymmetric gauge theories with adjoint matter," Phys. Lett. B 356, 492 (1995) [hep-th/9505088].

[39] R. G. Leigh and M. J. Strassler, "Exactly marginal operators and duality in fourdimensional N=1 supersymmetric gauge theory," Nucl. Phys. B 447, 95 (1995) [hepth/9503121].

[40] K. Landsteiner, E. Lopez and D. A. Lowe, "Duality of chiral N = 1 supersymmetric gauge theories via branes," JHEP 9802, 007 (1998) [hep-th/9801002].

[41] E. Lopez and B. Ormsby, "Duality for SU x SO and SU x Sp via branes," JHEP 9811, 020 (1998) [hep-th/9808125].

[42] A. Karch, D. Lust and G. Zoupanos, "Dualities in all order finite N = 1 gauge theories," Nucl. Phys. B 529, 96 (1998) [hep-th/9711157].

[43] P. Pouliot, "Chiral duals of nonchiral SUSY gauge theories," Phys. Lett. B 359, 108 (1995) [hep-th/9507018].

[44] P. Pouliot and M. J. Strassler, "A Chiral $S U(N)$ Gauge Theory and its Non-Chiral $\operatorname{Spin}(8)$ Dual," Phys. Lett. B 370, 76 (1996) [hep-th/9510228].

[45] P. Pouliot and M. J. Strassler, "Duality and Dynamical Supersymmetry Breaking in $\operatorname{Spin}(10)$ with a Spinor," Phys. Lett. B 375, 175 (1996) [hep-th/9602031]. 
[46] M. Berkooz, P. Cho, P. Kraus and M. J. Strassler, "Dual descriptions of SO(10) SUSY gauge theories with arbitrary numbers of spinors and vectors," Phys. Rev. D 56, 7166 (1997) [hep-th/9705003].

[47] T. Kawano, "Duality of N=1 Supersymmetric SO(10) Gauge Theory with Matter in the Spinorial Representation," Prog. Theor. Phys. 95, 963 (1996) [hep-th/9602035].

[48] P. Pouliot and M. Strassler, unpublished.

[49] P. Ramond, "Superalgebras in N = 1 gauge theories," Phys. Lett. B 390, 179 (1997) [hep-th/9608077].

[50] J. Distler and A. Karch, " $\mathrm{N}=1$ dualities for exceptional gauge groups and quantum global symmetries," Fortsch. Phys. 45, 517 (1997) [hep-th/9611088].

[51] A. Karch, "More on $\mathrm{N}=1$ self-dualities and exceptional gauge groups," Phys. Lett. B 405, 280 (1997) [hep-th/9702179].

[52] P. Cho, "Moduli in exceptional SUSY gauge theories," Phys. Rev. D 57, 5214 (1998) [hep-th/9712116].

[53] G. . 't Hooft, C. . Itzykson, A. . Jaffe, H. . Lehmann, P. K. Mitter, I. M. Singer and R. . Stora, "Recent Developments In Gauge Theories. Proceedings, Nato Advanced Study Institute, Cargese, France, August 26 - September 8, 1979," New York, Usa: Plenum (1980) 438 P. (Nato Advanced Study Institutes Series: Series B, Physics, 59).

[54] P. Pouliot, "Chirality and Duality," Seminar given at Caltech Theory Group, October 1996.

[55] J. H. Brodie, P. Cho and K. Intriligator, "Misleading anomaly matchings?," Phys. Lett. B 429, 319 (1998) [hep-th/9802092].

[56] H. Weyl, "The Classical Groups, Their Invariants and Representations," Princeton University Press, 1946.

[57] W. Fulton and J. Harris, "Representation Theory, A First Course," Springer-Verlag, 1991.

[58] P. Olver, "Classical Invariant Theory," London Mathematical Society Student Texts \#44, 1999.

[59] D. Mumford, J. Fogarty, F. Kirwan, "Geometric Invariant Theory," Third Edition, Springer-Verlag, 1994.

[60] B. Sturmfels, "Algorithms in Invariant Theory," Springer-Verlag, 1993. 
[61] V. L. Popov, "Groups, Generators, Syzygies, and Orbits in Invariant Theory," Translations of Mathematical Monographs, vol. 100, American Mathematical Society, 1992.

[62] J. Dieudonne and J. Carrell, "Invariant Theory, Old and New," Academic Press, 1971.

[63] R. Howe, "Perspectives on Invariant Theory," The Schur Lectures (1992), Israel Mathematical Conference Proceedings, 1995.

[64] G. Gurevich, "Foundations of the Theory of Algebraic Invariants," P. Noordhoff - Groningen, The Netherlands, 1964.

[65] G. Schwarz, "Invariant theory of $G_{2}$," Bull. Am. Math. Soc. 9, 335 (1983).

[66] G. Schwarz, "Invariant theory of $G_{2}$ and $\operatorname{Spin}_{7}$," Comment. Math. Helvetici 63, 624 (1988).

[67] G. Schwarz, "Representations of Simple Lie Groups with a Free Module of Covariants," Inventiones Math. 50, 1 (1978).

[68] G. Schwarz, "Representations of Simple Lie Groups with Regular Rings of Invariants," Inventiones Math. 49, 167 (1978).

[69] V. Popov, "Syzygies in the Theory of Invariants," Math. USSR Izvestiya 22, 507 (1984).

[70] Y. Gufan, Al. V. Popov, G. Sartori, V. Talamini, G. Valente and E. Vinberg, "Geometric Invariant Theory Approach to the Determination of Ground States D-wave Condensates in Isotropic Space," Jour. Math. Phys. 42, 1533 (2001).

[71] D. Kutasov, A. Schwimmer and N. Seiberg, "Chiral Rings, Singularity Theory and Electric-Magnetic Duality," Nucl. Phys. B 459, 455 (1996) [hep-th/9510222].

[72] P. Brax, C. Grojean and C. A. Savoy, "Anomaly matching and syzygies in N = 1 gauge theories," Nucl. Phys. B 561, 77 (1999) [hep-ph/9808345].

[73] P. Pouliot, "Molien function for duality," JHEP 9901, 021 (1999) [hep-th/9812015].

[74] F. Buccella, J. P. Derendinger, S. Ferrara and C. A. Savoy, "Patterns Of Symmetry Breaking In Supersymmetric Gauge Theories," Phys. Lett. B 115, 375 (1982).

[75] C. Procesi and G. W. Schwarz, "The Geometry Of Orbit Spaces And Gauge Symmetry Breaking In Supersymmetric Gauge Theories," Phys. Lett. B 161 (1985) 117.

[76] R. Gatto and G. Sartori, "Consequences Of The Complex Character Of The Internal Symmetry In Supersymmetric Theories," Commun. Math. Phys. 109, 327 (1987).

[77] M. A. Luty and W. I. Taylor, "Varieties of vacua in classical supersymmetric gauge theories," Phys. Rev. D 53, 3399 (1996) [hep-th/9506098]. 
[78] T. Gherghetta, C. Kolda and S. P. Martin, "Flat directions in the scalar potential of the supersymmetric standard model," Nucl. Phys. B 468, 37 (1996) [hep-ph/9510370].

[79] P. Brax and C. A. Savoy, "Supersymmetric flat directions and analytic gauge invariants," hep-th/0104077.

[80] C. Csaki and H. Murayama, "Discrete anomaly matching," Nucl. Phys. B 515, 114 (1998) [hep-th/9710105].

[81] P. C. Argyres, M. Ronen Plesser and N. Seiberg, "The Moduli Space of N=2 SUSY QCD and Duality in N=1 SUSY QCD," Nucl. Phys. B 471, 159 (1996) [hep-th/9603042].

[82] M. Berkooz, "A Comment on Non-Chiral Operators in SQCD and its Dual," Nucl. Phys. B 466, 75 (1996) [hep-th/9512024].

[83] M. van Leeuwen, A. Cohen and B. Lisser, "LiE Manual," version 2.1, Amsterdam, The Netherlands, 1996. 